\title{
First synthesis of wind-profiler signals on the basis of large-eddy simulation data
}

\author{
A. Muschinski, ${ }^{1}$ P. P. Sullivan, ${ }^{2}$ D. B. Wuertz, ${ }^{3}$ R. J. Hill,${ }^{3}$ S. A. Cohn,${ }^{4}$ \\ D. H. Lenschow, ${ }^{2}$ and R. J. Doviak ${ }^{5}$
}

\begin{abstract}
Radar wind profilers detect scatter from clear-air refractive-index irregularities. The Doppler shift calculated from the time series of the backscattered signal provides an estimate of the radial velocity of the air within the radar's resolution volume. It is known that there are quite a number of effects that can give rise to intrinsic biases in these radial-velocity estimates. It is extremely difficult to unambiguously identify these biases on the basis of observations since it is impossible to independently probe the threedimensional, time-dependent fine structure of the relevant atmospheric variables (wind and refractive index) within the radar's resolution volume down to the relevant spatial and temporal scales associated with the scattering process itself. In this paper the large-eddy simulation (LES) technique is used to generate, with high spatial and temporal resolution (vertical resolution, $8 \mathrm{~m}$; horizontal resolution, $16 \mathrm{~m}$; time step, $0.8 \mathrm{~s}$ ), data of atmospheric variables within a convective boundary layer. The LES data that are used in this study do not have the spatiotemporal resolution that would be needed for a deterministic, full solution of the scattering integral. However, a parameterized model of the scattering integral can be constructed on the basis of Kolmogorov-scaling arguments and a randomphase model. This model can be numerically solved on the basis of LES data. For the first time, LES-generated fields of virtual temperature, humidity, and three-dimensional wind are used to construct time series of the real and imaginary parts of wind-profiler signals. The simulated wind-profiler signals are processed to derive Doppler velocities, and the simulated Doppler velocities are compared with the (simulated) meteorological "in situ truth" provided directly by the LES wind-velocity data set. Examples of simulation results and the potential of the LES-based, wind-profiler simulation technique as well as some limitations are discussed.
\end{abstract}

\section{Introduction}

Radar wind profilers can "see" returns from refractive-index irregularities in clear air. VHF wind profilers typically operate at frequencies close to $50 \mathrm{MHz}$, which corresponds to wavelengths close to $6 \mathrm{~m}$. At these wavelengths the clear-air echoes typically dominate over cosmic noise, intrinsic system noise, and

\footnotetext{
${ }^{1}$ Cooperative Institute for Research in Environmental Sciences, University of Colorado/NOAA, and NOAA Environmental Technology Laboratory, Boulder, Colorado.

2 NCAR Mesoscale and Microscale Meteorology Division, Boulder, Colorado.

${ }^{3}$ NOAA Environmental Technology Laboratory, Boulder, Colorado.

${ }^{4}$ NCAR Atmospheric Technology Division, Boulder, Colorado.

${ }^{5}$ NOAA National Severe Storms Laboratory, Norman, Oklahoma.
}

Copyright 1999 by the American Geophysical Union.

Paper number 1999RS900090.

0048-6604/99/1999RS900090\$11.00 other echoes, for example, those from aerosols, insects, cloud droplets, and raindrops. At the shorter UHF wavelengths (typically 30 and $75 \mathrm{~cm}$, corresponding to frequencies of $1 \mathrm{GHz}$ and $400 \mathrm{MHz}$, respectively), Rayleigh scatter from raindrops can dominate over the clear-air echo, requiring sophisticated signal-processing techniques to distinguish between precipitation and clear-air signals, if possible. Wind profilers detect scatter from spatial refractiveindex irregularities with wavelengths of half the radar wavelength, the "Bragg wavelength." In wave number space the so-called "sampling function" has a sharp maximum at the "Bragg wave number"

$$
k_{B}=\frac{4 \pi}{\lambda}
$$

where $\lambda$ is the radar wavelength [e.g., Doviak and Zmić, 1984, 1993; Muschinski, 1998]. That is, as long as Rayleigh scatter does not play a role, wind profilers see only refractive-index structures at the Bragg wave 
number. While UHF wind profilers do not have a preferred direction at which the temporally averaged echo intensity has a maximum, VHF wind-profiler echo intensities typically do show a pronounced maximum at vertical and nearly vertical beam-pointing directions. (Depending on their power-aperture product, VHF radars are divided into mesosphere-stratosphere-troposphere (MST) radars and stratospheretroposphere (ST) radars. The term MST radar is usually reserved for the most powerful VHF radars, while the less powerful ones are often referred to as ST radars.) This "VHF aspect sensitivity" was discovered by Röttger and Liu [1978] and Gage and Green [1978], and there is general agreement among researchers in the field that the VHF aspect sensitivity is the manifestation of a significant anisotropy in the spatial spectrum of the 3-m-scale, clear-air, refractiveindex irregularities in the free atmosphere. Correspondingly, the absence of a significant aspect sensitivity at UHF frequencies in the atmospheric boundary layer as well as in the free atmosphere is seen as evidence for isotropy of decimeter-scale, refractive-index irregularities throughout the atmosphere.

The term "wind profiler" indicates that the main purpose of this kind of radar is to measure (vertical) profiles of the wind (vector) above the radar site. This does not mean, however, that wind profilers can measure only wind. There is a large body of literature providing evidence that wind profilers also reveal information about other atmospheric properties like the refractive-index structure parameter $C_{n}^{2}$ [e.g., Tatarskii, 1961; VanZandt et al., 1978; Muschinski, 1997], turbulent energy dissipation rate $\varepsilon$ [e.g., Frisch and Clifford, 1974; Gage et al., 1980; Hocking, 1983; Weinstock, 1981; Cohn, 1995; Delage et al., 1997; Rao et al., 1997], the inner and outer scales of turbulence [e.g., Muschinski, 1997; Eaton and Nastrom, 1998; Gossard et al., 1998], and temperature [Gage and Green, 1982] and humidity [Gossard et al., 1999].

During the past years, more and more wind profilers have been deployed for operational and research use, and it has been shown that wind profilers provide reliable wind measurements for the initialization of numerical weather-forecasting models [e.g., Smith and Benjamin, 1993]. Since wind profilers can provide wind measurements with a temporal resolution down to several seconds and even less (at least in the atmospheric boundary layer), wind profilers have been used to measure momentum fluxes [e.g., Vincent and Reid, 1983; Chang et al., 1997; Rao et al., 1997] and, in combination with radio acoustic sounding systems (RASS), heat fluxes [e.g., Angevine et al., 1993; Lippmann et al., 1997].

It has been pointed out that the Doppler velocity,

$$
v_{D}=-\frac{\omega_{D}}{k_{B}}
$$

which is directly retrieved from the Doppler shift $\omega_{D}$, is not always a reliable estimate of the radial air velocity within the radar's resolution volume. Significant radial velocity biases can arise from the aspect sensitivity of VHF echoes observed at near-zenith beam-pointing directions [Hocking et al., 1986] and from a nonzero correlation between $C_{n}^{2}$ and the radial wind velocity in a field of vertically propagating gravity waves [Nastrom and VanZandt, 1994; Hoppe and Fritts, 1995]. More recently, it has been pointed out [Muschinski, 1996b] that the VHF aspect sensitivity in combination with a nonsymmetric layer-tiltingangle distribution caused by Kelvin-Helmholtz instability events can lead to a bias in long-term averages of the Doppler velocity observed with vertically pointing VHF radars.

In the daytime convective boundary layer, gravity waves and Kelvin-Helmholtz instability do not appear and can be excluded as being a possible reason for vertical-velocity biases. Using UHF wind profilers, Angevine [1997] has observed a downward bias in the measured vertical velocity in the daytime boundary layer. The magnitude of this bias is between 10 and 30 $\mathrm{cm} \mathrm{s}^{-1}$, and Angevine [1997] attributes it to scatterers like insects or other small targets, which have an effect on the wind-profiler spectra but usually cannot be identified.

There is usually no "in situ truth" against which wind-profiler measurements can be tested with the required accuracy. (Airborne and tower measurements have provided much evidence that wind-profiler measurements are generally reliable (see, for example, the review by Gossard [1990]). Airborne and tower measurements, however, are always "point measurements," while radar measurements always provide volume averages, which makes the interpretation of a comparison always difficult; second, it is practically almost impossible to operate an in situ probe within the radar resolution volume without severely contaminating the radar data.) It is therefore generally difficult to quantify sampling errors and biases such as mentioned above and to verify theoretical models of sampling errors and biases. The 
purpose of this paper is to present and discuss a new technique to simulate time series of UHF windprofiler signals. This simulation technique is a combination of the large-eddy simulation (LES) technique and radio wave scattering theory. It will be shown that in contrast to real-world experiments, simulation experiments based on this technique are free from meteorological, logistical, financial, and technical uncertainties that typically affect real-world field experiments. LES-based wind-profiler simulation experiments like those described in this paper are expected to give more detailed and more conclusive insight into specific aspects of the potential and limitations of boundary-layer, wind-profiler measurements than corresponding real-world field experiments. On the other hand, an LES is only a model of a real flow, and therefore one has to be careful about the extent to which LES model assumptions affect LES-based wind-profiler simulations.

The paper is organized as follows: Section 2 describes the setup of the LES, and examples of LESgenerated fields of atmospheric quantities are shown. Section 3 describes the new technique that we apply to generate time series of $915-\mathrm{MHz}$, wind-profiler signals on the basis of LES data. In section 4, statistical properties of the simulated wind-profiler, vertical-wind observations and statistical properties of the LES-generated vertical-wind field are analyzed. The results are discussed in section 5. Section 6 gives a summary and conclusions.

\section{Large-Eddy Simulation (LES)}

\subsection{Suitability of LES for Modeling Clear-Air Radar Backscattering}

Today's supercomputers have large memories (typically a gigaword) and fast processors (typically gigaflops) that make it possible to numerically integrate the equations of motion of turbulent fluids fourdimensionally, i.e., in space $(x, y, z)$ and time $(t)$. The numerical integration of the Navier-Stokes equations is called "direct numerical simulation" (DNS). A DNS requires the grid spacing $\Delta$ to be comparable to or smaller than the Kolmogorov length,

$$
\eta=\left(\frac{\nu^{3}}{\varepsilon}\right)^{1 / 4},
$$

which gives the order of magnitude of the size of the smallest turbulent eddies. In (3), $\varepsilon$ is the turbulent energy dissipation rate, and $\nu$ is the molecular kine- matic viscosity. At length scales comparable to $\eta$, inertial forces and viscous forces are of the same order of magnitude. In the convective atmospheric boundary layer (CBL), $\eta$ is typically of the order of 1 $\mathrm{cm}$. Since the inversion height of the CBL is of the order of $1 \mathrm{~km}$, a DNS of a cubical volume of $1 \mathrm{~km}^{3}$ CBL would require of the order of $\left(10^{5}\right)^{3}=10^{15}$ grid points, which is many orders of magnitude beyond the capacity of even the most powerful contemporary computers. Today the biggest numerical grids in computer simulations of turbulent flows have of the order of 1000 grid points in each dimension. The order of magnitude of the ratio between the outer turbulent length scale $L$ and $\eta$ is set by the Reynolds number of the turbulent fluid:

$$
\operatorname{Re} \sim\left(\frac{L}{\eta}\right)^{3 / 4}
$$

Obviously, even when using the most powerful supercomputers, the DNS technique is limited to small or moderate values of Re. A DNS of the atmospheric CBL is beyond reach.

An efficient technique to reduce the required huge number of grid points is large-eddy simulation (LES). LES reduces the number of degrees of freedom by spatially filtering the Navier-Stokes equations [Lilly, 1967; Leonard, 1974]. The goal of LES is to resolve only the lower-wave-number part of the entire spectrum of turbulent fluctuations, which contains most of the variance of the fluctuating quantities. In other words, an LES resolves the "energy-containing range" of the turbulence but not the range in which most of the dissipation occurs. In the present study we use LES as a tool to four-dimensionally $(x, y, z, t)$ simulate local and instantaneous small-volume averages of turbulently fluctuating components of the velocity vector, as well as local and instantaneous values of the refractive-index structure parameter $C_{n}^{2}$, which characterizes the "subgrid-scale" inertial subrange of the refractive-index irregularities. The problem of how to conceptually deal with instantaneous small-volume averages of turbulently fluctuating quantities is very difficult and certainly touches the limits of what can be done rigorously with the tools of contemporary mathematics of random functions. In this study we heuristically assume that it is reasonable to allow $C_{n}^{2}$ to vary as a function of time and position [e.g., Tatarskii, 1961, section 4.3]. This generalization is suggested by a large body of obser- 
vational studies [e.g., Clifford, 1978]. We will come back to this issue in section 2.3 .

As an alternative to the traditional "subgrid-scale turbulence parameterization" concept [Lilly, 1967; Leonard, 1974], the LES equations may also be considered equations of motion for a certain class of hypothetical, non-Newtonian fluids [Muschinski, 1996a]. These "LES fluids" are designed such that their Kolmogorov length is fixed, while their (eddy) viscosity is variable. An important requirement when running an LES is that the grid spacing lie within the inertial subrange of the turbulence to be modeled, so that the effects of the subgrid (better "subfilter") turbulence, which is not resolved by the LES, on the resolved-scale flow can be formulated on the basis of the classical theory of fully developed, locally homogeneous, and isotropic turbulence. Muschinski [1996a] has discussed the LES concept from the traditional subgrid-scale turbulence parameterization viewpoint as well as on the basis of the LES fluids picture. The LES fluids concept makes it easier than the traditional "parameterization" concept to understand that an LES does not "know" of any random behavior at length scales smaller than and comparable to the grid spacing.

Since the pioneering studies by Lilly [1967] and Deardorff [1970], LES has been established as the technique of choice to model turbulent flows at high Reynolds numbers [e.g., Galperin and Orszag, 1993]. Boundary-layer meteorology, in particular, has benefitted significantly from the LES technique [e.g., Deardorff, 1980; Schmidt and Schumann, 1989; Kaltenbach et al., 1994; Mason, 1994; Peltier and Wyngaard, 1995; Dörnbrack, 1997; Sullivan et al., 1998; Wyngaard et al., 1998].

In order to employ the LES technique for the simulation of wind-profiler signals, one has to make sure that the fields of the three-dimensional wind vector and of the refractive index within the radar's resolution volume are resolved at least to a certain degree. The typical dimension of a UHF wind-profiler's resolution volume in the middle boundary layer is $100 \mathrm{~m}$, and the typical dwell time (the length of the time series from which a Doppler spectrum is computed) is of the order of $1 \mathrm{~s}$. (As has been pointed out by one of the reviewers, there is some ambiguity in the term "dwell time." Some authors define the dwell time as we defined it here (the length of the time series from which a fast Fourier transform (FFT) is computed), while others define it as the time during which the radar beam dwells in the same beam direction. The latter time is typically much longer than the dwell time according to our definition, because typically many spectra are measured and then incoherently averaged before the beam direction is changed. At this point, we do not feel a need to coin a new term for the length of the time series from which an FFT is computed. Instead, we assume that there is no incoherent averaging in our case, such that the two definitions of dwell time coincide.) If the LES's grid spacing is $10 \mathrm{~m}$, one would have of the order of $10^{3}$ LES grid cells per radar resolution volume.

A crucial point, which we will discuss in some detail, is the fact that a wind profiler sees irregularities at length scales of half the radar wavelength. The Bragg wavelength,

$$
L_{B}=\frac{\lambda}{2},
$$

is $16 \mathrm{~cm}$ for a standard $915-\mathrm{MHz}$ wind profiler. The grid spacing of a typical contemporary LES of the atmospheric CBL amounts to ten or a few tens of meters, which is about 2 orders of magnitude larger than the Bragg wavelength of a typical UHF wind profiler. Therefore a full, time-dependent solution of the scattering integral for UHF clear-air backscatter on the basis of a "conventional" LES of the turbulent refractive-index field is currently impossible. In section 3 we will describe a parameterization which we use to "bridge" the gap between a small Bragg wavelength and a comparatively large LES grid spacing.

There have been previous techniques to simulate atmospheric radar signals [e.g., Zrnić, 1975; May et al., 1989; Sheppard and Larsen, 1992; Holdsworth and Reid, 1995]. The LES-based simulation of clear-air radar signals that we describe in this paper has a major advantage over those previous studies. Namely, an LES relies on a spatially filtered surrogate of the Navier-Stokes equations, which allows one to realistically and self-consistently simulate structure and evolution of variances, covariances (particularly fluxes), and structure parameters of turbulently fluctuating atmospheric variables like the three-dimensional wind vector, temperature, humidity, and refractive index.

\subsection{The Setup of the LES}

We set up an LES similar to that described by Sullivan et al. [1996]. The number of grid points is 192 in all three directions. The grid spacing is $\Delta_{x}=\Delta_{y}=$ 
Table 1. Overview of the Large-Eddy Simulation Setup

\begin{tabular}{ll}
\hline \multicolumn{1}{c}{ Property } & Specification \\
\hline Number of grid points & $192^{3}$ \\
Horizontal resolution & $16 \mathrm{~m}$ \\
Vertical resolution & $8 \mathrm{~m}$ \\
Size of the model domain & $3072 \times 3072 \times 1536 \mathrm{~m}$ \\
Prognostic variables & $u, v, w, E, \Theta, s_{1}, s_{2}$ \\
Surface flux of sensible heat & $240 \mathrm{~W} \mathrm{~m}$ \\
Inversion height & $900 \mathrm{~m}$ \\
Inversion strength & $2 \mathrm{~K}$ \\
\hline
\end{tabular}

$16 \mathrm{~m}$ in the horizontal directions and $\Delta_{z}=8 \mathrm{~m}$ in the vertical direction. The size of the model domain is $3.072 \mathrm{~km}$ in the $x$ and $y$ directions and $1.536 \mathrm{~km}$ in the vertical direction.

The variables simulated by the LES are the two horizontal wind velocity components, $u$ and $v$, the vertical velocity component $w$, the subfilter turbulent kinetic energy $E$, the virtual potential temperature $\Theta$, and two passive scalars, $s_{1}$ and $s_{2}$. The scalar $s_{1}$ has a source at the ground and a sink above the inversion. We rescale $s_{1}$ such that we obtain a specific humidity $q$, with statistical properties in space and time consistent with the characteristics of the other quantities. The overall characteristics of the simulated CBL are as follows: The inversion height is about $900 \mathrm{~m}$, and the surface flux of sensible heat is $240 \mathrm{~W} \mathrm{~m}^{-2}$. Table 1 gives an overview of the most important characteristics of the LES setup. Plate 1 shows horizontal cross sections from an instantaneous three-dimensional, vertical-velocity field simulated by the LES. Plate 1a shows $w(x, y)$ close to the ground, where a cell structure resembling Rayleigh-Benard convection is clearly visible [cf. Schmidt and Schumann, 1989], and Plate $1 \mathrm{~b}$ shows $w(x, y)$ at an altitude just below the inversion.

\subsection{Calculating a $C_{n}^{2}$ Field From LES-Generated Fields of Temperature and Humidity}

Figure 1 shows vertical profiles of horizontally averaged fields of potential temperature $\Theta$ and specific humidity $q$ obtained from the same LES realization as shown in Plate 1 . One clearly identifies the boundary-layer inversion at about $900 \mathrm{~m}$, where $\Theta$ increases rapidly with height and where $q$ drops from its mixed-layer value to almost zero. Through the gas equations for dry air and water vapor, the specific humidity $q$ is related to the water vapor pressure $p_{w}$ :

$$
p_{w}=1.608 q p,
$$

where $p$ is total pressure.

In order to simulate wind-profiler signals that result from Bragg scattering from clear-air, refractive-index irregularities, we need to generate a field of the refractive-index turbulence structure parameter, $C_{n}^{2}$. The refractive index $n$ is calculated as follows:

$$
n=c_{d} \frac{p}{\Theta}+c_{w} \frac{p_{w}}{\Theta^{2}}
$$

where $c_{d}=7.76 \times 10^{-7} \mathrm{~K} \mathrm{~Pa}^{-1}$ and $c_{w}=3.73 \times$ $10^{-3} \mathrm{~K}^{2} \mathrm{~Pa}^{-1}$ are constants [e.g., Doviak and Zrnić, 1993, pp. 16 and 466]. Like Peltier and Wyngaard [1995], we ignore the effects of variations of the air pressure $p$ on the variations of $n$ and compute variations of $n$ from variations in the potential temperature $\Theta$ and from variations of $q$. We neglect the very small difference between fluctuations of the potential temperature and of the actual temperature in the boundary layer. These are reasonable assumptions if the CBL is comparatively shallow, as in the present study, and if the Mach number is small compared to unity.

There are different ways to retrieve $C_{n}^{2}$ from data of $n$. The most direct way is to use the defining equation

$$
C_{n}^{2}=\frac{\left\langle[n(\mathbf{r}+\mathbf{\delta})-n(\mathbf{r})]^{2}\right\rangle_{\mathbf{r}}}{|\mathbf{\delta}|^{2 / 3}}
$$

Here \langle\rangle$_{\mathbf{r}}$ denotes the spatial average over a volume within which the $n$ irregularities are assumed to be statistically isotropic and homogeneous. Here $\mathbf{r}$ is the position vector, and $\boldsymbol{\delta}$ is the vector defining a spatial separation. An LES simulates values of locally averaged variables as a function of space and time. That is, variables like $u, v, w$, and $n$ are not simulated as point values but as spatial averages over volumes the size of which are determined by the grid spacing and by the eddy-viscosity parameterization that is used within the LES. This property of the LES-generated data allows us to use local gradients simulated by the LES to diagnostically compute local and instantaneous structure parameters.

In the LES that we use in this study the variables are staggered such that the nodes of the scalars are located vertically between nodes of the vertical velocity. We calculate the field of the refractive-index turbulence structure parameter $C_{n}^{2}$ diagnostically from the $n$ field as follows: 

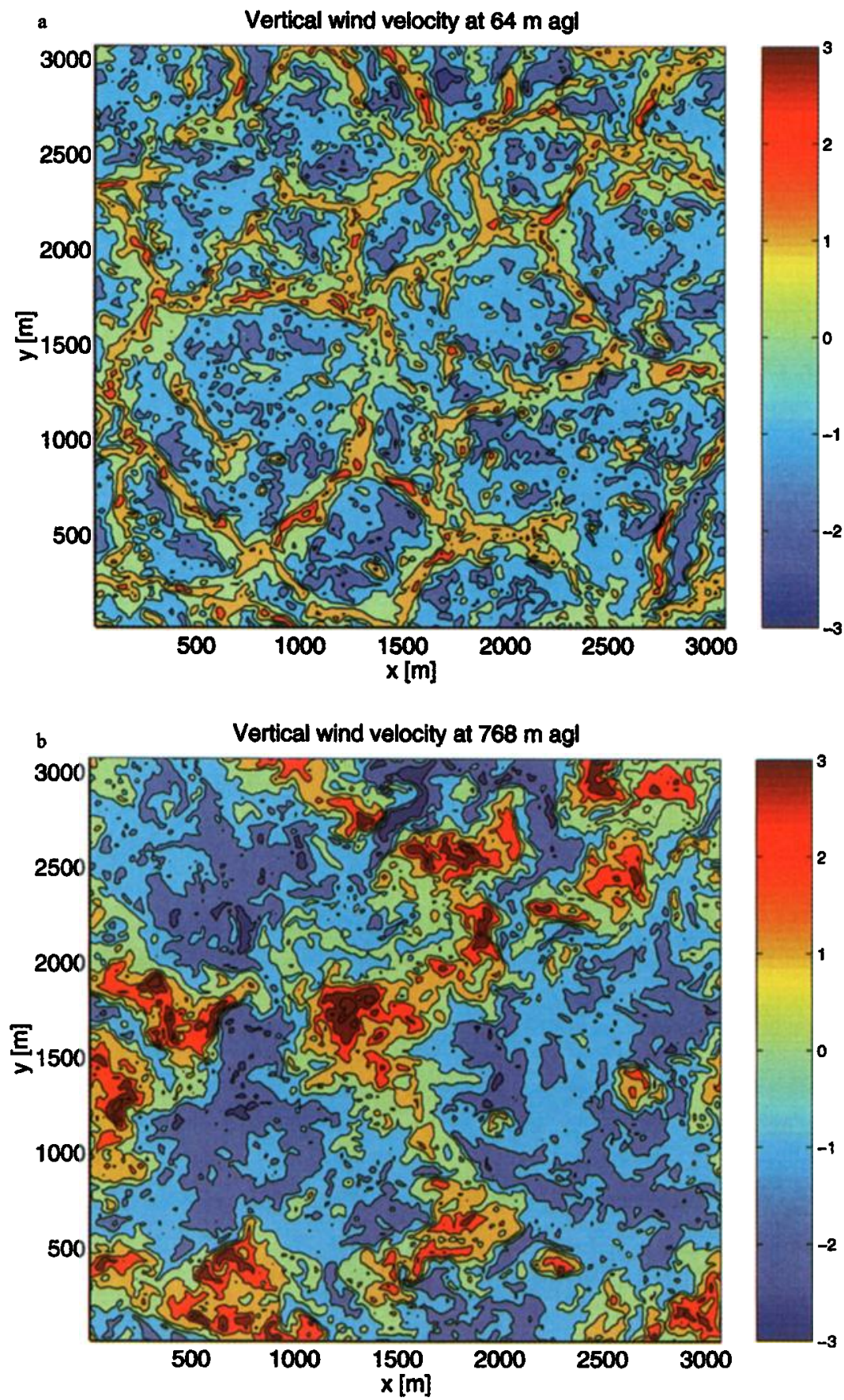

Plate 1. Horizontal cross sections of an instantaneous, three-dimensional, vertical-velocity field predicted by the large-eddy simulation (LES): (a) $w(x, y)$ at $64 \mathrm{~m}$ above ground level, and (b) $w(x, y)$ at $768 \mathrm{~m}$ above ground level. The cell structure close to the ground does not exist in the upper regions of the convective atmospheric boundary layer (CBL). The velocities at the color bars are given in units of $\mathrm{ms}^{-1}$. 

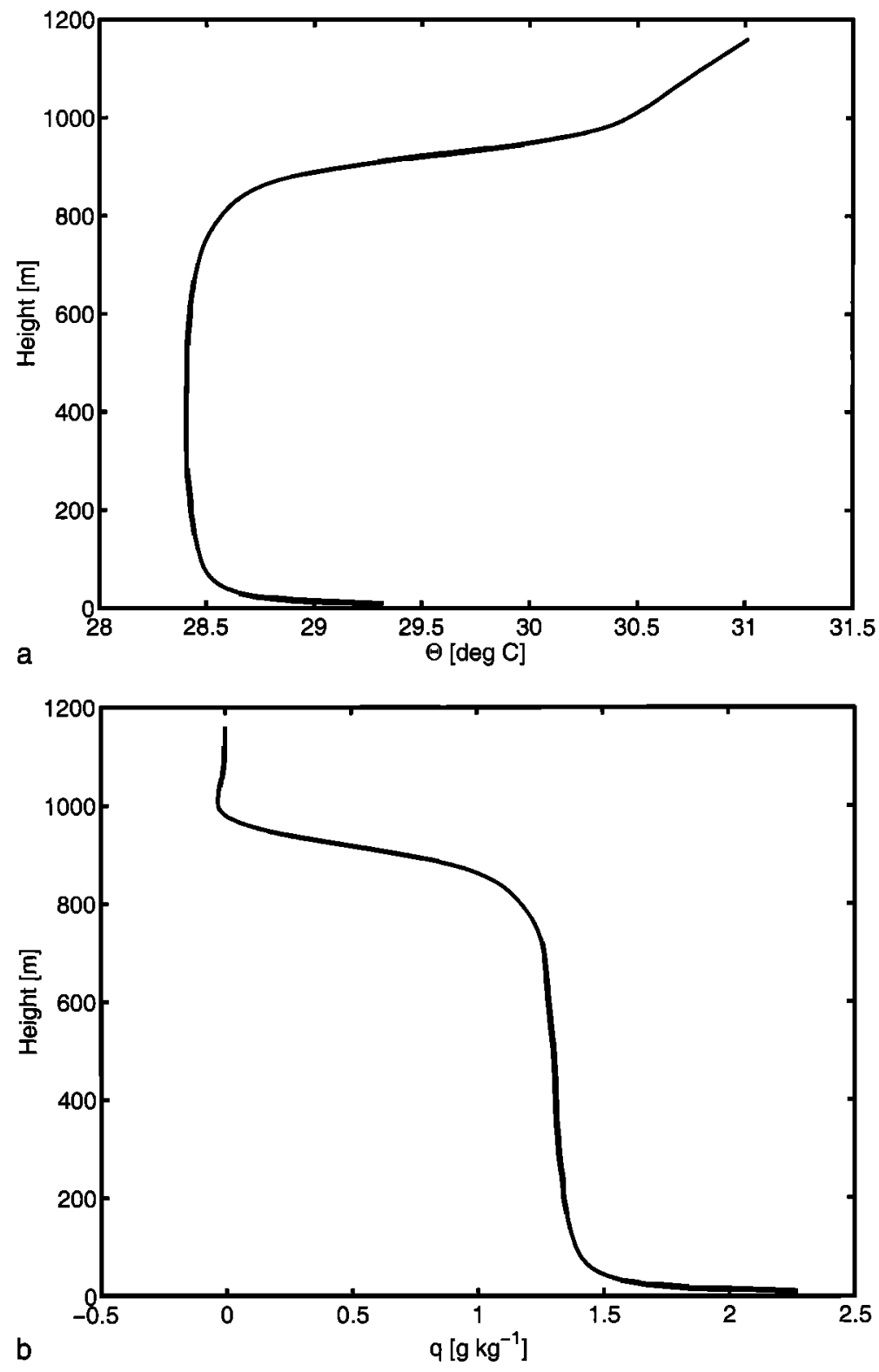

Figure 1. Horizontally averaged vertical profiles of quantities characterizing the CBL, obtained from a single realization: (a) virtual potential temperature, and (b) specific humidity.

$$
\begin{aligned}
& C_{n}^{2}(x, y, z, t) \\
& \quad=\frac{\left[n\left(x, y, z+\frac{\Delta_{z}}{2}, t\right)-n\left(x, y, z-\frac{\Delta_{z}}{2}, t\right)\right]^{2}}{\Delta_{z}^{2 / 3}},
\end{aligned}
$$

where $\Delta_{z}$ is the grid spacing in the vertical direction.

Using (9), a three-dimensional (3-D) field of local and instantaneous values of $C_{n}^{2}$ is computed on the basis of a 3-D field of local and instantaneous LESpredicted gradients of $n$, in full analogy to the Smagorinsky-Lilly parameterization [Lilly, 1967], which diagnostically provides local and instantaneous values of eddy viscosity and energy dissipation rate on the basis of local and instantaneous values of the magnitude of the three-dimensional shear vector evaluated at individual LES grid cells.

Two advantages of (9) are that first, it makes full 


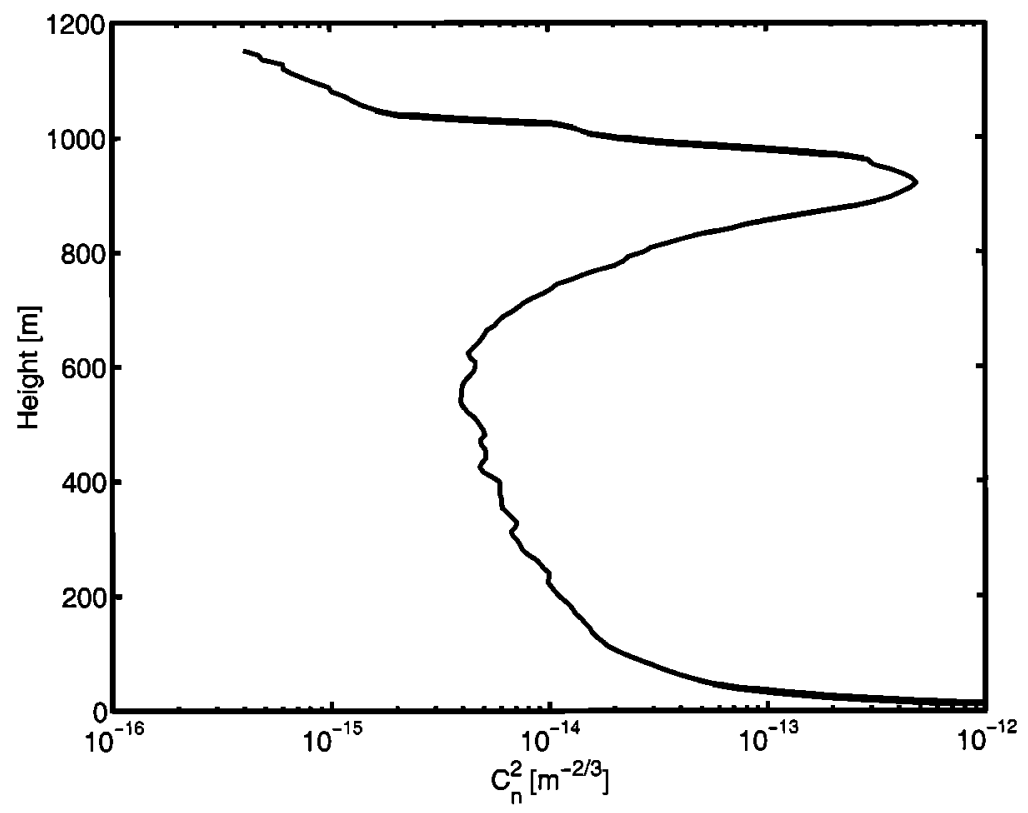

Figure 2. Vertical profile of the horizontally averaged refractive-index structure parameter $C_{n}^{2}$ within the LES-generated CBL, obtained from a single realization in time.

use of the high resolution $(8 \mathrm{~m})$ in the vertical direction, and second, it provides a $C_{n}^{2}$ value for each $w$ node. (If one would compute $C_{n}^{2}$ values from horizontal spacings, the width of the smallest centered interval would be $32 \mathrm{~m}$, instead of $8 \mathrm{~m}$ in the vertical direction.) A possible disadvantage of estimating $C_{n}^{2}$ values from differences squares of $n$ across distances of $\Delta_{z}$, instead of across distances of $2 \Delta_{x}$, is that there might be a systematic underestimation of $C_{n}^{2}$ because the finite sharpness of the LESinherent spatial filter has a damping effect on the turbulent fluctuations at the smallest resolvable length scales [e.g., Muschinski, 1996a; Mason and Brown, 1999]. In our study, however, because the LES-generated $C_{n}^{2}$ fields are by the model assumptions consistent with the volume reflectivity that the simulated radar observes, an underestimation of $C_{n}^{2}$ by a constant factor of the order of unity would not play any role for our purposes.

Figure 2 shows the vertical profile of the horizontal averages of the local $C_{n}^{2}$ values obtained from (9). Without going into detail at this point, we can say that this profile of $C_{n}^{2}$ qualitatively agrees, as expected, with previous large-eddy simulations [e.g., Peltier and Wyngaard, 1995] and with observations (see, for example, the overview by Doviak and Zrnić [1993, pp. 469-475]).

\section{Simulation of Wind-Profiler Signals on the Basis of an LES Data Set}

In this section, which is the central part of this paper, we describe how time series of wind-profiler signals can be constructed on the basis of an LES data set. First, we point out that CBL data simulated with a contemporary LES are too coarse to allow a direct numerical integration of the scattering integral; that is, the LES data contain no phase information at length scales comparable to the Bragg wavelength. Second, we describe how to parameterize scattering amplitudes and phases on the basis of quantities predicted by the LES. Third, we investigate how the phases within a simulated radar resolution volume change with time during a period smaller than or comparable to the LES time step. Fourth, we present exemplary, simulated time series of clear-air radar signals and their Doppler spectra, and we briefly discuss their statistical properties. Fifth, we consider theoretically the spatial structure function of the temporal phase changes within the radar's resolution volume, and we define a phase-correlation timescale. We show the conditions under which the spectral broadening of the returns from refractive-index irregularities within individual LES grid cells in the radar resolution volume may be neglected. 


\subsection{No Phase Information From the LES Data}

In contrast to in situ measurement systems, a wind profiler uses a very indirect method to probe the atmosphere: Electromagnetic pulses are transmitted into the atmosphere; a very small part of the electromagnetic energy is scattered or reflected back to the radar; and a time series of phase-coherent complex signals, $I(t)$, is recorded, which can be written as a spatial integral (the "scattering integral"):

$$
I(t)=A \int W(\mathbf{r}) n^{\prime}(\mathbf{r}, t) e^{-\jmath k_{B} r_{t}} d^{3} r,
$$

[see, e.g., Doviak and Zrnić, 1984]. In (10), $A$ is a constant,

$$
A=\frac{g}{\lambda r_{0}^{2}}\left(\frac{P_{t}}{2 R}\right)^{1 / 2},
$$

where $g$ is the antenna gain, $\lambda$ is the radar wavelength, $r_{0}$ is the range (the distance between the antenna center and the center of the radar's resolution volume under consideration), $P_{t}$ is the transmitted power, and $R$ is the receiver resistance. Other variables in (10) are the radar's three-dimensional one-way weighting function $W(\mathbf{r})$, and the field of refractive-index irregularities $n^{\prime}(\mathbf{r}, t)$ within the radar's resolution volume at the time $t$. The phase factor $\exp \left(-j k_{B} r_{t}\right)$ is defined by the Bragg wave number $k_{B}=4 \pi / \lambda$ and the distance $r_{t}$ between the integration point $\mathbf{r}$ and the antenna center. Because $\exp \left(-j k_{B} r_{t}\right)$ oscillates rapidly within the radar resolution volume (the volume in which the weighting function $W(\mathbf{r})$ has values that are large enough to contribute significantly to the scattering integral), a full numerical integration of the scattering integral would require $n^{\prime}(\mathbf{r}, t)$ be known with a spatial resolution of about a tenth of $\lambda$. In other words, the grid spacing has to be so small that $\exp \left(-j k_{B} r_{t}\right)$ can be represented numerically. This would require

$$
\Delta \ll \lambda,
$$

a condition that is not fulfilled by contemporary LES of the atmospheric CBL if $\lambda$ is in the UHF range. Instead, we have to deal with the opposite case:

$$
\Delta \gg \lambda \text {. }
$$

So how can we numerically integrate the scattering integral on the basis of a data set of $n^{\prime}(\mathbf{r}, t)$ that is about 2 orders of magnitude too coarse to fulfill the condition given in (12)?

\subsection{Parameterization of Phases and Amplitudes}

The underlying idea of LES is that the subfilter turbulence is locally homogeneous and isotropic. Because in our case the Bragg wavelength is much smaller than $\Delta$, this automatically implies that the LES-generated turbulent fields are isotropic at the Bragg wave number. In the following, we will see that this model assumption considerably simplifies the problem of how to construct clear-air wind-profiler signals on the basis of LES data.

An integral over a domain can be written as a sum of integrals over subdomains that fill the domain. Consider the $N$ LES grid cells that cover the radar resolution volume, i.e., the region wherein $W(\mathbf{r})$ is nonnegligible. We write the scattering integral (10) as a sum of contributions from those LES grid-cell subvolumes $V_{p}$ :

$$
I(t)=A \sum_{p=1}^{N} \int_{V_{p}} W(\mathbf{r}) n^{\prime}(\mathbf{r}, t) e^{-j k_{B} r_{t}} d^{3} r .
$$

Assuming that the subdomains $V_{p}$ (i.e., the LES grid cells) are much smaller than the radar resolution volume, such that $W(\mathbf{r})$ may be considered constant within one LES grid cell, we introduce

$$
W_{p}=W\left(\mathbf{r}_{p}\right),
$$

where $\mathbf{r}_{p}$ is the position of the center of the $p$ th LES grid cell out of the $N$ grid cells over which the numerical integration of the scattering integral is carried out. Then we can rewrite (14) as follows:

$$
I(t)=\sum_{p=1}^{N} I_{p}(t)=A \sum_{p=1}^{N} W_{p} a_{p}(t) e^{-\jmath \varphi_{p}(t)}
$$

That is, $A W_{p} a_{p}$ is the magnitude and $\varphi_{p}$ is the phase of the contribution $I_{p}$ to the total backscattered signal I.

We assume that the different phases $\varphi_{p}$ are statistically independent from each other. If there were a finite correlation between any two phases $\varphi_{1}$ and $\varphi_{2}$, then the ensemble average of the sum of $I_{1}$ and $I_{2}$ would sensitively depend on the exact positions (within a fraction of the Bragg wavelength) of the centers of the LES grid cells, which cells are about 100 times larger than the Bragg wavelength. This would make no sense, and we conclude that the phases must be assumed to be statistically independent. On the basis of this assumption, we obtain the following ensemble average of the backscattered power: 


$$
\begin{aligned}
\left\langle P_{r}\right\rangle & =\left\langle\frac{R}{2} I^{*} I\right\rangle \\
& =\left\langle\frac{R}{2} A^{2}\left(\sum_{p=1}^{N} W_{p} a_{p} e^{+J \varphi_{p}}\right)\left(\sum_{q=1}^{N} W_{q} a_{q} e^{-j \varphi_{q}}\right)\right\rangle \\
& =\left\langle\frac{R}{2} A^{2} \sum_{p=1}^{N} \sum_{q=1}^{N} W_{p} a_{p} e^{+J \varphi_{p}} W_{q} a_{q} e^{-j \varphi_{q}}\right\rangle \\
& =\frac{R}{2} A^{2} \sum_{p=1}^{N} W_{p}^{2} a_{p}^{2},
\end{aligned}
$$

where the angle brackets denote the average over the ensemble of statistically independent phases.

On the other hand, the backscattered power can be written as a weighted spatial integral over the localized, spatial refractive-index variance spectrum $\Phi_{n}(\mathbf{r}$, $\mathbf{k})$, taken at the Bragg wave number $k_{B}$ at which $\Phi_{n}(\mathbf{r}, \mathbf{k})$ is assumed to be isotropic:

$$
\begin{aligned}
\left\langle P_{r}\right\rangle & =\frac{R}{2} A^{2} \iiint W^{2}(\mathbf{r}) \Phi_{n}\left(\mathbf{r}, k_{B}\right) d^{3} r \\
& =\frac{R}{2} A^{2} \sum_{p=1}^{N} W_{p}^{2}\left[\Phi_{n}\left(k_{B}\right)\right]_{p} V_{p}
\end{aligned}
$$

where we have assumed that $W^{2}(\mathbf{r})$ and $\Phi_{n}\left(\mathbf{r}, k_{B}\right)$ vary sufficiently smoothly with position $\mathbf{r}$ [see, e.g., Tatarskii, 1961, section 4.3; Muschinski, 1998, sections 3.2 and 6.1].

Then we can make use of the well-known relationship between $\Phi_{n}\left(k_{B}\right)$ and $C_{n}^{2}$ [Tatarskii, 1961, equation (3.52)], which we interpret locally, i.e., which we assume to be valid within the volume $V_{p}$ centered at $\mathbf{r}_{p}:$

$$
\begin{aligned}
{\left[\Phi_{n}\left(k_{B}\right)\right]_{p} } & =\frac{\Gamma\left(\frac{8}{3}\right) \sin \left(\frac{\pi}{3}\right)}{4 \pi^{2}}\left[C_{n}^{2}\right]_{p} k_{B}^{-11 / 3} \\
& =0.0330\left[C_{n}^{2}\right]_{p} k_{B}^{-11 / 3} .
\end{aligned}
$$

After combining (17), (18), and (19), we obtain the following equation, which allows us to simulate a wind-profiler signal on the basis of $C_{n}^{2}$ fields and changes in the phases $\varphi_{p}$ obtained from LES data:
$I(t)=A \sqrt{0.0330 k_{B}^{-11 / 3}} \sum_{p=1}^{N} W_{p} \sqrt{\left[C_{n}^{2}(t)\right]_{p} V_{p}} e^{-J \varphi_{p}(t)}$.

That is, the amplitudes $a_{p}\left(t_{0}\right)$ at some initial time $t_{0}$ are given by

$$
a_{p}\left(t_{0}\right)=\sqrt{\left[C_{n}^{2}\left(t_{0}\right)\right]_{p} V_{p}}
$$

\subsection{Phase Shifts With Time}

Assuming that we know the initial amplitudes $a_{p}\left(t_{0}\right)$, and assuming that the phases $\varphi_{p}$ are statistically independent from each other, we have to think about how to simulate the temporal changes of phases and amplitudes during the dwell time. The dwell time $T_{d}$ is operationally defined as the length of the radar signal time series from which a (nonaveraged) Doppler spectrum is computed. The coherentintegration time $\Delta t$ is the temporal spacing between two adjacent points in the time series. (Usually, it is important to carefully distinguish between the pulse repetition period and the "coherent-integration time" $\Delta t$. The coherent-integration time is the period of time during which the individual echo samples are phase-coherently averaged, i.e., integrated. Typically, tens or hundreds of echo samples are coherently integrated. The two main reasons for coherent integration are to improve the signal-to-noise ratio and to reduce the amount of data that need to be Fourierprocessed. In our simulation, however, we leave problems related to a finite signal-to-noise ratio out of account and assume that a noise-free signal $I$ is sampled at the sampling frequency $1 / \Delta t$.)

In this paper we show how time series of windprofiler signals can be simulated on the basis of a single LES "snapshot," i.e., on the basis of a single realization of the fields of three-dimensional wind and of refractive index. (At the end of this section we will describe how longer radar time series could be constructed.) We have set up the LES in a way that the time step is $0.8 \mathrm{~s}$. This means that we may assume that the fields of wind and refractive index (therefore also of $C_{n}^{2}$ ) are sufficiently stationary for $0.8 \mathrm{~s}$. For our simulation the dwell time has to be long enough to provide a reasonable spectral resolution for a typical Doppler spectrum obtained from boundarylayer signals, but it also must be short enough to avoid problems associated with nonstationarity of the LESpredicted wind- and refractive-index fields during the dwell time.

Let us assume that during the dwell time $T_{d}$, the 
refractive-index irregularities within an LES grid cell are advected in any direction with a velocity $\mathbf{v}$. Then the vector of the advection path is $\mathbf{d}=\mathbf{v} T_{d}$. Since we allow $C_{n}^{2}$ and the wind vector to vary from grid cell to grid cell, the assumption of stationarity during the period $T_{d}$ becomes questionable if components of $\mathbf{d}$ are comparable to or even larger than the LES grid spacings in the respective directions. This leads to a condition for $T_{d}$ :

$$
T_{d}<\min \left\{\frac{\Delta_{x}}{u} ; \frac{\Delta_{y}}{v} ; \frac{\Delta_{z}}{w}\right\} .
$$

This is simultaneously the Courant-Friedrichs-Lewy (CFL) criterion for the maximum time step of the LES. The CFL criterion has to be fulfilled in order to guarantee numerical stability during an LES run. We conclude that the LES time step is automatically a good choice for the maximum dwell time of the wind-profiler simulation if the signal is to be constructed on the basis of a single "LES time slice," i.e., on the basis of LES-generated fields obtained at a single time step. (This is what we do throughout this paper.) In our case the vertical grid spacing is $8 \mathrm{~m}$. Assuming a (vertical) wind velocity of a few meters per second, (22) requires $T_{d}$ to be smaller than a few seconds. We choose a dwell time of $T_{d}=1 \mathrm{~s}$. During $1 \mathrm{~s}$ an air parcel travels a few meters, which is of the order of 10 Bragg wavelengths if the wavelength is $32.8 \mathrm{~cm}$. This is sufficient to simulate a signal that has a sufficient number of oscillations during the sampling interval, so that Fourier processing would provide a statistically significant value for the Doppler shift. We choose a coherent-integration time $\Delta t$ of $1 / 128 \mathrm{~s}=7.8 \mathrm{~ms}$.

We assume that during $T_{d}$ the refractive-index irregularity field within the $p$ th LES grid cell is "frozen" and advected with the local and instantaneous velocity vector $\mathbf{v}_{p}$. This is a weak version of Taylor's [1938] "frozen turbulence" hypothesis. That is, we neglect changes of local $C_{n}^{2}$ during $T_{d}$.

Now we introduce the Bragg wave vector $\mathbf{k}_{B}^{(p)}$ as the vector that is directed from the antenna center to the center of the $p$ th LES grid cell and has the length $k_{B}$. That is, after a time $\tau$, which we assume to be smaller than $T_{d}$, the phase $\varphi_{p}$ has shifted by the amount

$$
\Delta \varphi_{p}=\mathbf{k}_{B}^{(p)} \cdot \mathbf{v}_{p}\left(t_{0}\right) \tau
$$

That is,
$\varphi_{p}\left(t_{0}+\tau\right)=\varphi_{0}^{(p)}+\Delta \varphi_{p}(\tau)=\varphi_{0}^{(p)}+\mathbf{k}_{B}^{(p)} \cdot \mathbf{v}_{p}\left(t_{0}\right) \tau$.

From (16) and (24) we obtain a simple formula that we can use to simulate time series of wind-profiler signals on the basis of a single LES snapshot:

$$
\begin{gathered}
I\left(t_{0}+\tau\right)=A^{\prime} \sum_{p=1}^{N} W_{p} \sqrt{\left[C_{n}^{2}\left(t_{0}\right)\right]_{p} V_{p}} \\
\cdot \exp \left[-j\left(\varphi_{0}^{(p)}+\mathbf{k}_{B}^{(p)} \cdot \mathbf{v}_{p}\left(t_{0}\right) \tau\right)\right]
\end{gathered}
$$

where the new constant $A^{\prime}$ is proportional to $A$,

$$
A^{\prime}=A \sqrt{0.0330} k_{B}^{-11 / 6} .
$$

In this study we restrict ourselves to dwell times that are not much longer than the LES time step, so that (24), which assumes that the phases of the individual Bragg scatterers change linearly with time, is a good approximation. It is straightforward, however, to generalize the concept to construct signal time series that are much longer than the LES time step: First, a temporal sequence of LES-generated fields of $C_{n}^{2}$ and the three velocity components $u, v$, and $w$ is selected. The temporal resolution of this sequence is equal to the LES time step, which typically is much larger than the coherent-integration time of a wind profiler. Second, one interpolates for each LES grid cell within the radar resolution volume the time series of $C_{n}^{2}, u, v$, and $w$, such that each interpolated time series has a temporal resolution defined by the coherent-integration time of the radar that is to be simulated. The interpolation smoothes temporal discontinuities in the temporal rate of phase change, $\partial \varphi / \partial t$, at times when there are switches between two subsequent LES fields. Third, a random set of initial phases $\varphi_{0}^{(p)}$ is generated, and for each LES grid cell a time series of the phase $\varphi_{p}(t)$ is computed by temporally integrating the phase changes that are obtained from the interpolated time series of $u, v$, and $w$. Fourth, a generalized version of (25) is used to generate an arbitrarily long time series of $I$ :

$$
I(t)=A^{\prime} \sum_{p=1}^{N} W_{p} \sqrt{\left[C_{n}^{2}(t)\right]_{p} V_{p}} \exp \left[-j \varphi_{p}(t)\right] .
$$

However, there is a limitation to application of (27) that is analogous to the CFL criterion (22) which limits application of (25). The reason for that limita- 

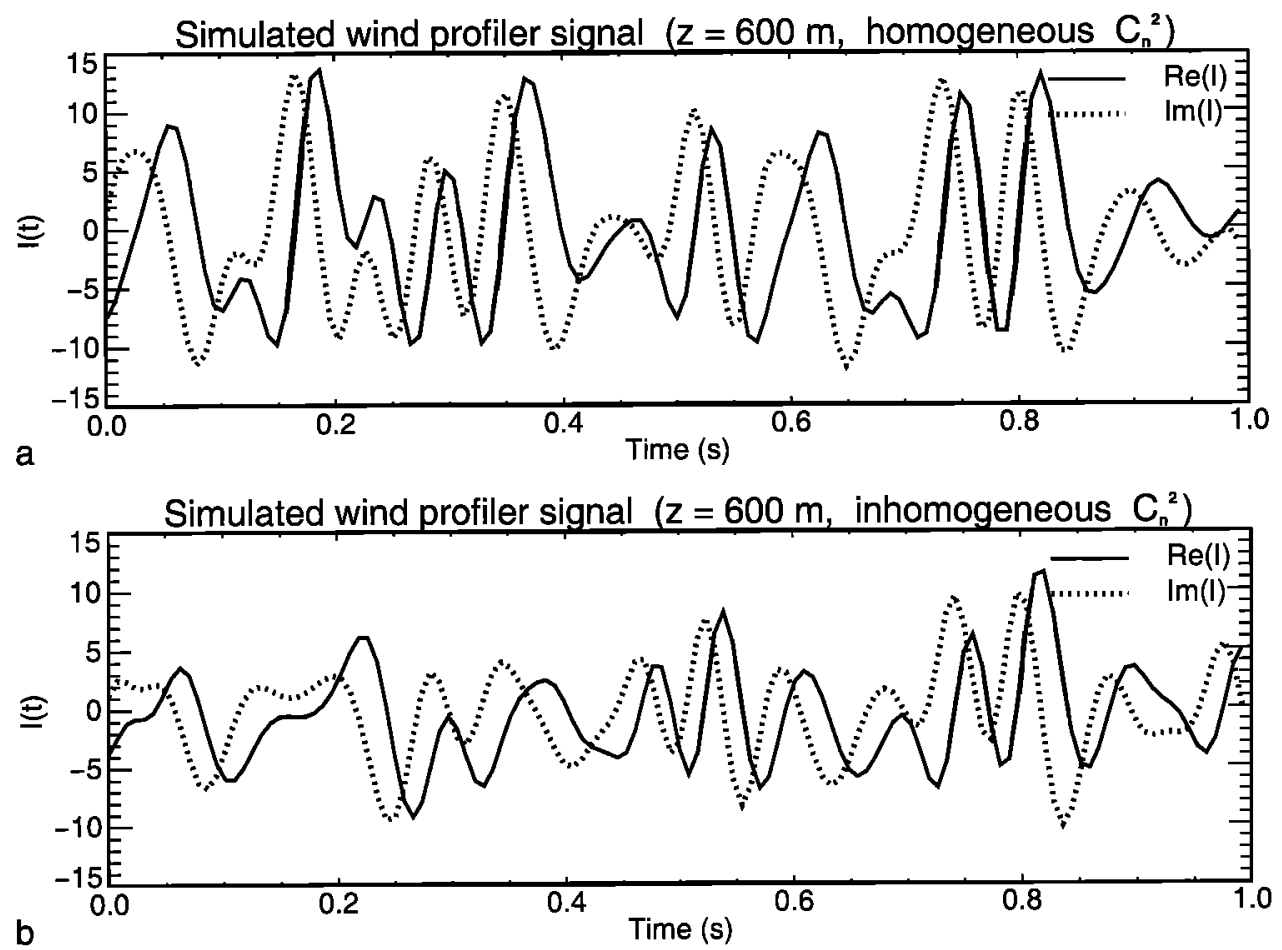

Figure 3. An example for simulated time series of a wind-profiler signal $I(t)$ obtained at a range of $600 \mathrm{~m}$. The solid lines represent the real part of $I(t)$, and the dotted lines are the imaginary part of $I(t)$. (a) Simulation assuming homogeneous $C_{n}^{2}$. (b) Simulation assuming the instantaneous (inhomogeneous) field of $C_{n}^{2}$ retrieved from the LES data.

tion is that (27) has been derived based on the assumption that each individual Bragg scatterer (i.e., the parcel of turbulent air within a respective LES grid cell) behaves like a point scatterer with infinite lifetime. For a given energy dissipation rate $\varepsilon$, however, the rms velocity within a grid cell is of the order of $(\varepsilon \Delta)^{1 / 3}$, so that within the grid cell is the refractiveindex irregularity pattern at the Bragg length scale $\lambda / 2$ is reorganized after a timescale of the order of $\lambda /(\varepsilon \Delta)^{1 / 3}$. That is, a Bragg scatterer has lost its phase coherency after a time comparable to $\lambda /(\varepsilon \Delta)^{1 / 3}$, and for proper use of (27) it may be necessary to randomly change the phases of the Bragg scatterers in time intervals of the order of $\lambda /(\varepsilon \Delta)^{1 / 3}$. We will come back to the issue of the finite lifetime of a Bragg scatterer in section 3.5, where we discuss the effect of spectral broadening due to turbulent motion at length scales that are not resolved by the LES.

\subsection{Doppler Spectrum and Radial Velocity Histogram}

Figure 3 shows, as an example, two time series of the real and imaginary part of a clear-air radar signal simulated by using (25). In Figure $3 a$ we have assumed that $C_{n}^{2}$ is constant within the pulse volume; in Figure 3b, however, we have used the field of $C_{n}^{2}$ within the radar resolution volume as obtained from (9), and we have assumed that the field of $C_{n}^{2}$ is stationary during the dwell time of $1 \mathrm{~s}$. The initial phases $\varphi_{0}^{(p)}$ have been assumed to be statistically independent, i.e., they have been randomly generated by assuming a probability density function that is uniform between $-\pi$ and $\pi$ and that is zero everywhere else.

Figure 4 shows as thin lines the power spectra of the two signals shown in Figure 3. Figure 4a corresponds to Figure $3 b$, and Figure $4 b$ corresponds to Figure $3 \mathrm{~b}$. Additionally, histograms of the properly rescaled, LES-generated, radial velocities $\left(v_{r}\right)$ are shown as bold lines in Figure 4. In Figure 4a the histogram of the $W^{2}(\mathbf{r})$-weighted, spatial $v_{r}$ distribution is shown, and Figure $4 \mathrm{~b}$ shows the histogram of the $C_{n}^{2}(\mathbf{r}) W^{2}(\mathbf{r})$-weighted $v_{r}$ distribution, corresponding to the spectra which have been simulated with homogeneous and inhomogeneous $C_{n}^{2}$ distribu- 

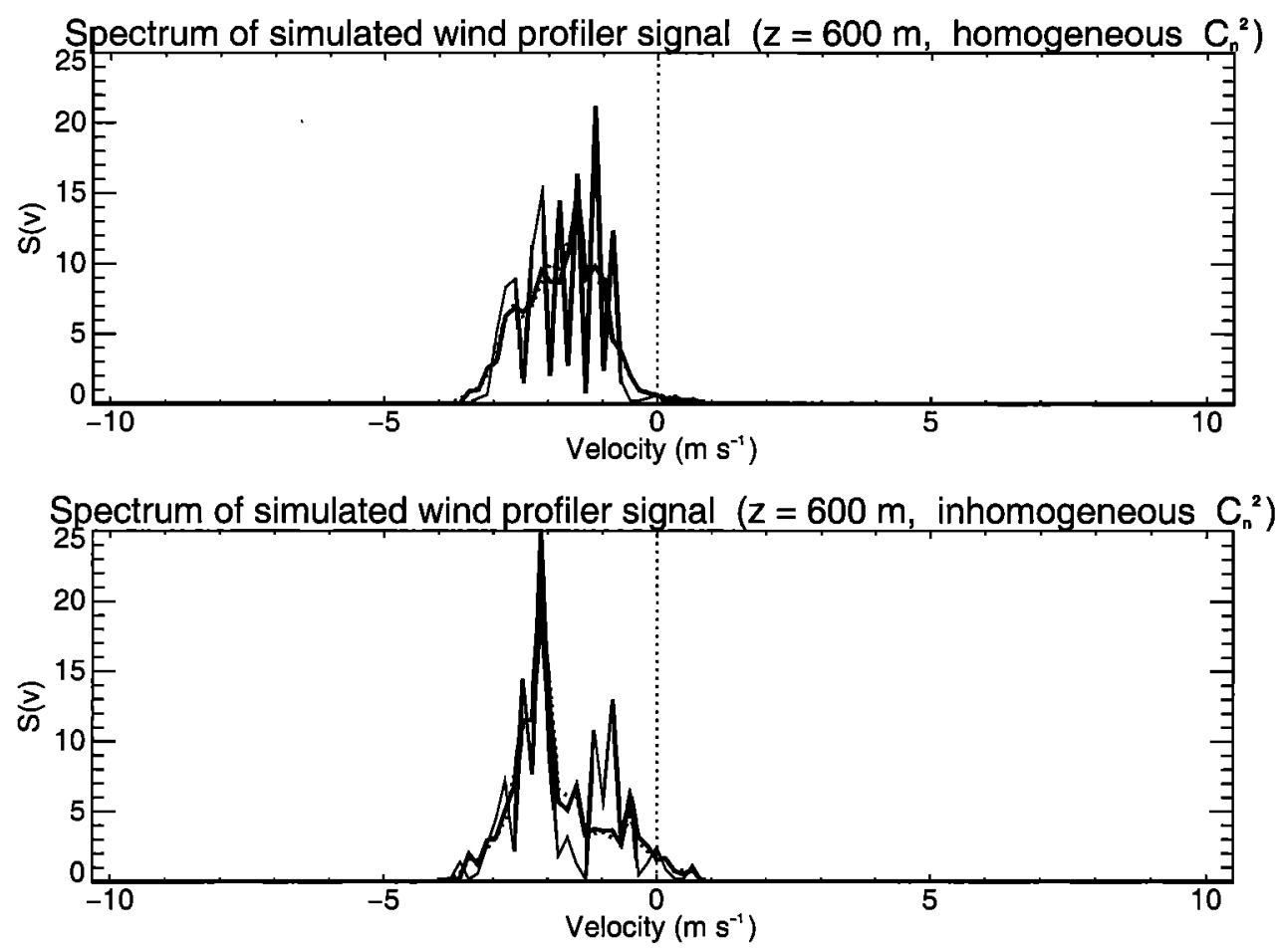

Figure 4. The thin lines represent the spectra of the two simulated wind-profiler time series shown in Figure 3. The bold lines are properly weighted and properly rescaled histograms of the radial-velocity distributions generated by the LES. The dotted lines are ensemble-averaged Doppler spectra obtained from 1000 realizations of signal time series, one of which is shown in Figure 3. The 1000 realizations of $I(t)$ have been obtained from the same LES fields but from 1000 independent sets of initial phases. As expected, there is good agreement between the ensemble-averaged Doppler spectra and the corresponding radial-velocity histograms.

tions, respectively. The $v_{r}$ histograms have been normalized such that the integrals over the Doppler spectrum and the integral over the respective histogram are equal to each other.

It is clearly seen that the histograms are much smoother than the spectra. The Doppler spectra are "spiky." This is to be expected since the values of nonsmoothed and nonaveraged Doppler spectra at a specific frequency $\omega$ (or within a very narrow frequency band) are exponentially distributed, i.e., they obey a chi-square distribution with two degrees of freedom (the real part and imaginary part of the Fourier transform) [see, e.g., Blackman and Tukey, 1958, pp. 21ff.; Jenkins and Watts, 1968, pp. 79 ff.]. In an exponentially distributed ensemble the standard deviation is equal to the mean [see, e.g., Jenkins and Watts, 1968, p. 70]. See also the appendix, where we give a mathematical analysis of the ensemble-averaged Doppler spectrum on the basis of the "random phases" assumption. As can be seen in Figure 4, the rms differences between the spectral estimates and the histogram estimates amount to about the histogram estimates themselves, which is in agreement with both the analysis in appendix and the expectation that the simulated spectral estimates are exponentially distributed.

The dotted lines in Figures $4 \mathrm{a}$ and $4 \mathrm{~b}$ are ensembleaveraged Doppler spectra: One thousand independent sets of initial phases $\varphi_{0}^{(p)}$ were randomly generated; for each set of $\varphi_{0}^{(p)}$ a signal time series $I(t)$ was computed using (25); a spiky spectrum of each realization of $I(t)$ was calculated, and the 1000 individual spectra were averaged. The two ensemble-averaged Doppler spectra (dotted lines) agree well with the respective histograms (bold solid lines). As a consistency check, we calculated also the two ensemble averages of the Doppler spectrum in the limit of an infinite number of realizations by using (A10) and 


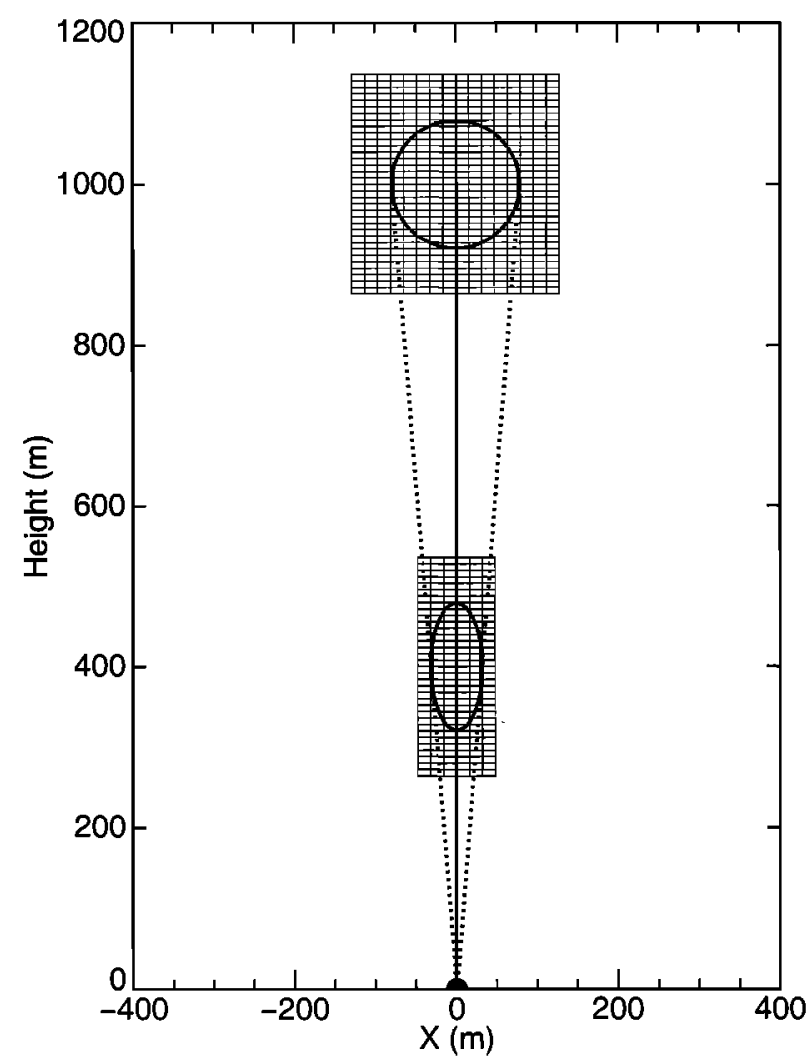

Figure 5. Size and geometry of the subvolumes of the LES model domain that are used to simulate wind-profiler signals for different radar resolution volumes. The grids represent the horizontal and vertical resolution of the LES data. The ellipses symbolize the half-power surfaces for the radar resolution volumes centered at 400 and $1000 \mathrm{~m}$ altitude. The LES subdomain centered at $1000 \mathrm{~m}$ altitude contains as many as 10,115 grid points.

(A11). The resulting graphs are not distinguishable from the histograms (bold solid lines in Figure 4), as expected.

Figure 5 shows size and geometry of the simulated wind profiler's resolution volumes centered at 400 and $1000 \mathrm{~m}$ altitude, as well as of the LES subdomains that were used to simulate the two wind-profiler signals. (The only reason to introduce the LES subdomains was to reduce the computational expense for numerically solving the scattering integral. We have taken care that the LES subdomains span regions sufficiently large so that the truncation of $W(\mathbf{r})$, which has been assumed to be a three-dimensional Gaussian function, is negligible.) The grids represent the horizontal and vertical resolution of 16 and $8 \mathrm{~m}$, respectively. The ellipses symbolize the half-power contour
Table 2. Wind-Profiler Specifications

\begin{tabular}{ll}
\hline \multicolumn{1}{c}{ Quantity } & \multicolumn{1}{c}{ Value } \\
\hline Frequency & $915 \mathrm{MHz}$ \\
Wavelength & $32.8 \mathrm{~cm}$ \\
Full half-power beam width & $9^{\circ}$ \\
Pulse duration & $1 \mu \mathrm{s}$ \\
Range resolution & $150 \mathrm{~m}$ \\
Pulse-volume width at $1 \mathrm{~km}$ range & $156 \mathrm{~m}$ \\
Dwell time & $1 \mathrm{~s}$ \\
Coherent integration time & $7.8 \mathrm{~ms}$ \\
Number of fast Fourier transform points per spectrum & 128 \\
\hline
\end{tabular}

lines of the radar's weighting functions that are associated with the radar resolution volumes centered at the respective altitudes. The LES subdomain centered at $1000 \mathrm{~m}$ altitude contains 10,115 LES grid cells (17 grid points in the horizontal directions and 35 grid points along the vertical direction). At lower altitudes the LES subdomains are smaller because of the smaller horizontal width of the pulse volumes at those altitudes. Table 2 gives a summary of the characteristics of the wind profiler that we have simulated.

\subsection{The Structure Function of the Rate of Change of Phase Differences: Spectral Broadening Due to Scales of Turbulence Unresolved by the LES}

Consider two small volume elements $d V_{1}$ and $d V_{2}$ within the radar resolution volume. At the moment, we do not make an assumption on whether $d V_{1}$ and $d V_{2}$ are in the same LES grid cell or in different LES grid cells.

Let the phases of $d I_{1}$ and $d I_{2}$ (the contributions to the scattering integral associated with $d V_{1}$ and $d V_{2}$, respectively) at any instant of time be $\varphi_{1}$ and $\varphi_{2}$, respectively. Because of the spatially rapidly oscillating phase factors $e^{-j k_{B} r_{1}}$ and $e^{-j k_{B} r_{2}}$, where $r_{1}$ and $r_{2}$ are the distances from the antenna center to $d V_{1}$ and $d V_{2}$, respectively, the phases $\varphi_{1}$ and $\varphi_{2}$ depend sensitively on $r_{1}$ and $r_{2}$, respectively.

If in a gedankenexperiment we would shift the volume elements $d V_{1}$ and $d V_{2}$ by amounts of the order of $\lambda / 4$ in radial directions, the phases $\varphi_{1}$ and $\varphi_{2}$ would be shifted by amounts of the order of $\pi$, so we could make $d I_{1}$ and $d I_{2}$ statistically independent by randomly shifting $d V_{1}$ and $d V_{2}$ radially by distances of the order of $\lambda / 4$. There would be no statistical independence, however, if we would carry out the random translations parallel to a surface of constant phase, i.e., in any direction transverse to the vector that points from the antenna center to $d V_{1}$ and $d V_{2}$. 
Now we investigate in more detail how the phase difference

$$
\Delta \varphi=\varphi_{2}-\varphi_{1}
$$

between $d I_{1}$ and $d I_{2}$ changes with time and how its temporal rate of change depends on the distance between the two subvolumes $d V_{1}$ and $d V_{2}$. Let $\delta=$ $\mathbf{r}_{2}-\mathbf{r}_{1}$ be the vector that points from $d V_{1}$ to $d V_{2}$. Then, (25) provides the phase difference at the time $t_{0}+\tau$, where $t_{0}$ is the beginning of the dwell time:

$$
\Delta \varphi=\Delta \varphi\left(t_{0}\right)+\mathbf{k}_{B}^{(2)} \cdot \mathbf{v}_{2} \tau-\mathbf{k}_{B}^{(1)} \cdot \mathbf{v}_{1} \tau .
$$

If the radar's beam width is not too large and if the magnitude of $\boldsymbol{\delta}$ is not larger than the radar resolution volume, conditions that are fulfilled in the case that we are interested in, then $\mathbf{k}_{B}^{(1)}$ is nearly parallel to $\mathbf{k}_{B}^{(2)}$, and we have

$$
\Delta \varphi=\Delta \varphi\left(t_{0}\right)+\mathbf{k}_{B} \cdot \Delta \mathbf{v}(\boldsymbol{\delta}) \tau,
$$

where $\mathbf{k}_{B}$ is a vector with length $k_{B}$ pointing from the antenna along the (vertically pointing) radar beam axis and $\Delta \mathbf{v}=\mathbf{v}_{2}-\mathbf{v}_{1}$ is the vector difference of the velocities in the volumes $d V_{1}$ and $d V_{2}$. Obviously, the rate of change of the phase difference is

$$
\frac{\partial}{\partial \tau} \Delta \varphi(\mathbf{\delta})=\mathbf{k}_{B} \cdot \Delta \mathbf{v}(\mathbf{\delta})
$$

Now we consider the variance (i.e., the ensemble average of the square) of $(\partial / \partial \tau) \Delta \varphi$ as a function of the separation $\mathbf{\delta}$; that is, we consider the spatial structure function of the temporal rate of phase change:

$$
\begin{aligned}
D_{\partial \varphi / \partial \tau}(\boldsymbol{\delta}) & =\left\langle\left(\frac{\partial}{\partial \tau} \Delta \varphi(\boldsymbol{\delta})\right)^{2}\right\rangle=\left\langle\left[\mathbf{k}_{B} \cdot \Delta \mathbf{v}(\boldsymbol{\delta})\right]^{2}\right\rangle \\
& =k_{B}\left\langle[\Delta w(\boldsymbol{\delta})]^{2}\right\rangle .
\end{aligned}
$$

In the case of isotropic turbulence we have

$$
\left\langle[\Delta w(\delta)]^{2}\right\rangle=\beta(\varepsilon \delta)^{2 / 3},
$$

where $\varepsilon$ is the energy dissipation rate, $\delta$ is the magnitude of the separation vector $\delta$, and $\beta$ is a numerical coefficient that has a universal value of 2.0 if $\delta$ points vertically (longitudinally) and a universal value of $\frac{4}{3} 2.0 \approx 2.7$ if $\delta$ points horizontally (transverse) [e.g., Yaglom, 1981]. If $\boldsymbol{\delta}$ points in an intermediate direction, then $\beta$ has a value between 2.0 and 2.7. For the following estimates we assume an intermediate value of 2.4 :

$$
\begin{aligned}
\sqrt{D_{\partial \varphi / \partial \tau}(\boldsymbol{\delta})} & =k_{B} \sqrt{\beta}(\varepsilon \delta)^{1 / 3}=4 \pi \sqrt{2.4} \frac{(\varepsilon \delta)^{1 / 3}}{\lambda} \\
& =19.5 \frac{(\varepsilon \delta)^{1 / 3}}{\lambda}
\end{aligned}
$$

Now we define the "phase correlation timescale" $\tau_{\varphi}$ as the time that has to elapse until the rms phase difference has changed by the amount $\pi$ :

$\tau_{\varphi}(\delta)=\pi \sqrt{D_{\partial \varphi / \partial \tau}(\delta)}=\frac{1}{4 \sqrt{\beta}} \frac{\lambda}{(\varepsilon \delta)^{1 / 3}}=0.16 \frac{\lambda}{(\varepsilon \delta)^{1 / 3}}$.

That is, we find the same timescale, $\lambda /(\varepsilon \delta)^{1 / 3}$, that we have interpreted at the end of section 3.3 as the lifetime of a Bragg scatterer with a diameter of the order of $\delta$.

In the case of a 915-MHz wind profiler we have $\lambda=$ $32.8 \mathrm{~cm}$, and a typical value of the energy dissipation rate in the atmospheric boundary layer is $\varepsilon=10^{-3}$ $\mathrm{m}^{2} \mathrm{~s}^{-3}$. Therefore the phase factors of two Bragg scatterers separated by a distance $\delta=10 \mathrm{~m}$ (comparable to the grid spacing of the LES that we used for this study) are statistically independent after $0.24 \mathrm{~s}$. From $\tau_{\varphi}$ as a function of $\delta$ we obtain an estimate of how much is contributed to the broadening of the Doppler spectrum by the "subfilter turbulence," i.e., by the small-scale turbulence within an individual LES grid cell, which we neglect in our simulation. From (2) and (35) we know that the following contribution to the Doppler-spectrum width is caused by turbulent motion having length scales smaller than $\Delta$ :

$$
\Delta \sigma_{v}=\frac{2 \Delta \omega_{D}}{k_{B}}=\frac{2 \frac{\pi}{\tau_{\varphi}}}{k_{B}}=\frac{\lambda}{2 \tau_{\varphi}}=0.78(\varepsilon \Delta)^{1 / 3} .
$$

Now consider a radar resolution volume that has a maximum diameter $B$. If the outer length scale of the inertial range is not smaller than $B$, then the rms velocity within that volume, i.e., the total spectral width $\sigma_{v}$, is of the order of $(\varepsilon B)^{1 / 3}$, and then the ratio between the loss of spectral width due to the nonresolved subfilter turbulence and the total spectral width and is of the order of

$$
\frac{\Delta \sigma_{v}}{\sigma_{v}} \approx \frac{(\varepsilon \Delta)^{1 / 3}}{(\varepsilon B)^{1 / 3}}=\left(\frac{\Delta}{B}\right)^{1 / 3} .
$$

That is, the relative loss of spectral width is of the order of $1 / 10$ even if the LES grid spacing $\Delta$ were 
$10^{-3} B$ and therefore not negligible in simulations of the spectral width.

Inserting $\varepsilon=10^{-3} \mathrm{~m}^{2} \mathrm{~s}^{-3}$ and $\Delta=10 \mathrm{~m}$ in (36) leads to $\Delta \sigma_{v}=0.34 \mathrm{~m} \mathrm{~s}^{-1}$. The expression for $\Delta \sigma_{v}$ in (36) can also be formally compared against the resolution of $v_{r}$ due to a finite dwell time $T_{d}$ [e.g., Gossard and Strauch, 1983, equation 11-10]:

$$
\Delta v_{r}=\frac{\lambda}{2 T_{d}} .
$$

Comparing (36) and (38) leads to the conclusion that the (missing) spectral broadening due to the nonresolved LES subfilter turbulence is smaller than the spectral resolution of the simulated Doppler spectra if the dwell time $T_{d}$ (the length of the time series from which the spectrum is computed) is smaller than the phase correlation time $\tau_{\varphi}$. If the spectra are computed from longer simulated time series, the increased spectral resolution of the simulated spectra must not be misinterpreted as providing more accurate simulations of the spectral width. If the simulation technique presented in this paper is used to simulate techniques to retrieve the energy dissipation rate $\varepsilon$ from the spectral width as proposed by Frisch and Clifford [1974], it has to be taken into account that the simulated spectral width does not contain the spectral broadening by the subfilter turbulence, which is not resolved by the LES. We do not know, however, how to analytically correct for the "missing spectral broadening," because the effective spatial filter of an LES is not known [Muschinski, 1996a].

\section{Data Analysis}

In this section we analyze the LES data and the wind-profiler signals that we simulated on the basis of the LES data set.

\subsection{Single Doppler-Velocity Profiles}

Figure 6 shows five different vertical profiles of the vertical velocity $(w)$ above the grid point $(96,96,0)$. The thin solid line with triangles is an instantaneous vertical line profile of $w$, obtained from an instantaneous three-dimensional realization of $w(x, y, z)$ as generated by the LES. We refer to this profile as the "simulated true" (instantaneous) $w$ profile. (Throughout this paper we are dealing with simulations of radar measurements made during short dwell times, i.e., dwell times that are not larger than the LES time step. All profiles shown in this paper have been generated on the basis of the same LES realization.) It is assumed that the grid point $(96,96,0)$ is the site of the wind profiler the signals of which are simulated. The bold dashed line is the spatially averaged, simulated, true $w$ profile; that is, the simulated, true $w$ field has been three-dimensionally weighted with the wind profiler's three-dimensional power weighting function $W^{2}(\mathbf{r})$. The solid line is the $w$ field, weighted with $W^{2}(\mathbf{r}) C_{n}^{2}(\mathbf{r})$, where $C_{n}^{2}(\mathbf{r})$ is the instantaneous $C_{n}^{2}$ field as diagnostically calculated from the LES-generated fields by using (9). The squares and the crosses represent $v_{D}$ profiles, i.e., vertical profiles of the Doppler velocities obtained from the Doppler shifts of the wind-profiler signals simulated for the respective height, in steps of $\Delta_{z}$. The squares are Doppler velocities retrieved from wind-profiler signals that have been simulated assuming homogeneous $C_{n}^{2}$ within the respective radar resolution volume; the crosses are Doppler velocities retrieved from windprofiler signals that have been simulated assuming the instantaneous, inhomogeneous $C_{n}^{2}$ distribution as provided by the LES. The dash-dotted line is not a velocity profile but the vertical profile of the $W^{2}(\mathbf{r})$ weighted $C_{n}^{2}$ field. We may interpret this profile as a profile of the instantaneous radar reflectivity.

The $v_{D}$ profile simulated with homogeneous $C_{n}^{2}$ (squares) follows nicely the profile of the threedimensional, spatially averaged, true $w$ field (dashed line), and the $v_{D}$ profile simulated with inhomogeneous $C_{n}^{2}$ (crosses) is in good agreement with the vertical profile of the $W^{2}(\mathbf{r}) C_{n}^{2}(\mathbf{r})$-weighted, i.e., reflectivity-weighted $w$. There is a clear disagreement, though, between the $W^{2}(\mathbf{r})$-weighted $w$ profile and the $W^{2}(\mathbf{r}) C_{n}^{2}(\mathbf{r})$-weighted $w$ profile, and, correspondingly, between the $v_{D}$ profile simulated with homogeneous $C_{n}^{2}$ (squares) and the $v_{D}$ profile simulated with inhomogeneous $C_{n}^{2}$ (crosses). This disagreement clearly indicates that wind estimates based on the Doppler shift can be significantly biased if there is a large vertical gradient of the $W^{2}(\mathbf{r})$-weighted $C_{n}^{2}(\mathbf{r})$ during the observation. In a region where both $C_{n}^{2}$ and $w$ increase with height, the $W^{2}(\mathbf{r}) C_{n}^{2}(\mathbf{r})$-weighted $w$ is essentially the vertical velocity within the upper region of the respective radar resolution volume and not the vertical velocity at its center. That is, if both $C_{n}^{2}$ and $w$ increase with height, the $W^{2}(\mathbf{r}) C_{n}^{2}(\mathbf{r})$-weighted $w$ overestimates the true spatially averaged vertical velocity. In a region where $C_{n}^{2}$ increases and $w$ decreases with height, however, the $W^{2}(\mathbf{r})$-weighted $w$ is always smaller than the true, spatially averaged, vertical velocity, as can be seen in the region between 600 and $800 \mathrm{~m}$ in Figure 6. 


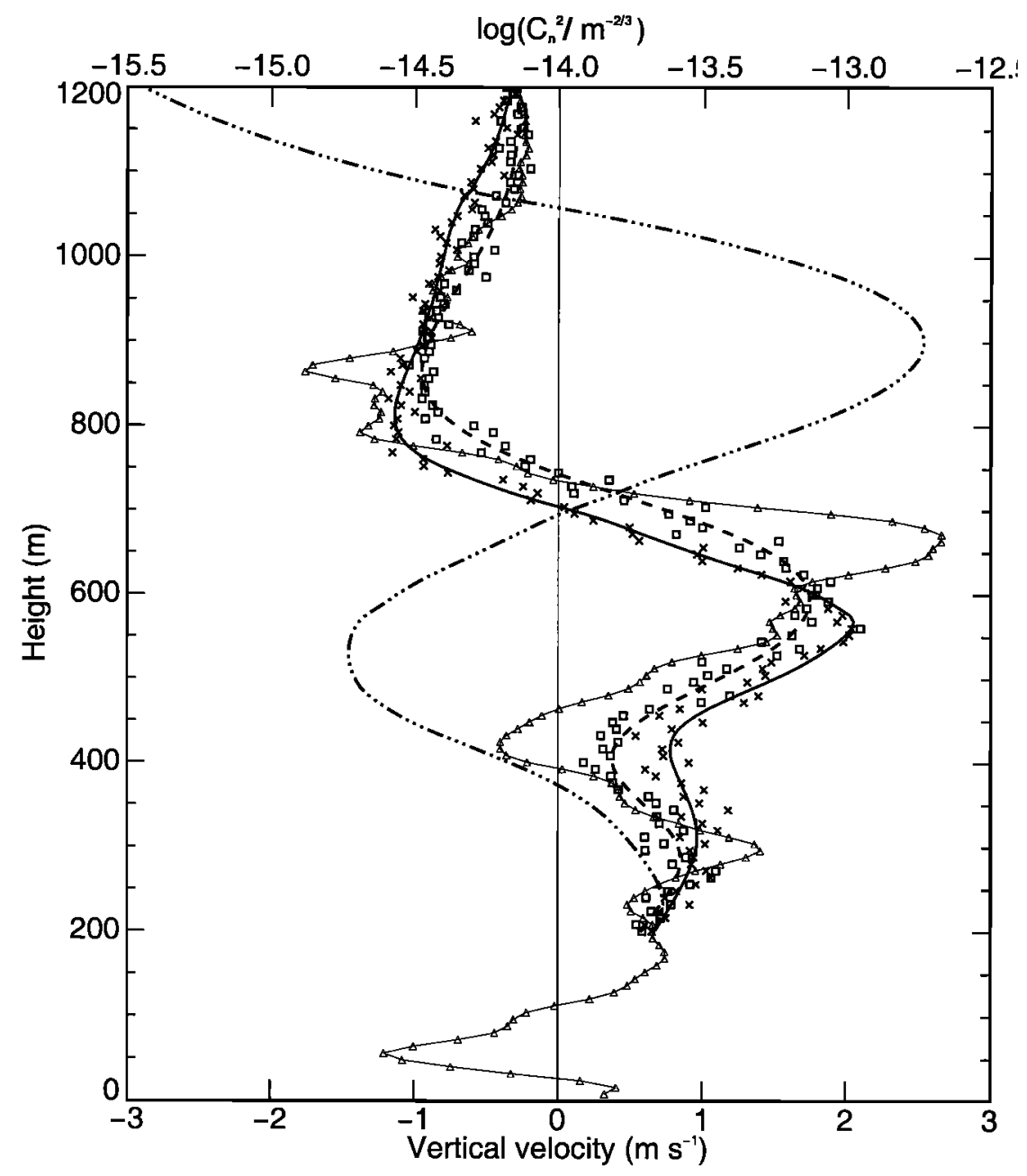

Figure 6. Vertical profiles of "simulated true" and "simulated observed" vertical wind $(w)$ in a simulated, convective, atmospheric boundary layer, generated with a large-eddy simulation (LES) model. Solid line with triangles designates LES-generated $w$ line profile above the site of the simulated wind profiler; dashed line designates vertical profile of LES-generated $w$, spatially weighted with the wind profiler's power weighting function $W^{2}$; solid line designates vertical profile of $w$, spatially weighted with the product of $W^{2}$ and $C_{n}^{2}$; squares designate Doppler velocities retrieved from the simulated radar signal time series, assuming uniform $C_{n}^{2}$; crosses designate Doppler velocities retrieved from the signal time series simulated on the basis of the LES-generated $C_{n}^{2}$-field; and dash-dotted line designates vertical profile of the $W^{2}$-weighted $C_{n}^{2}$.

\subsection{Vertical Profiles of Horizontal Averages Over an Array of $\mathbf{4 0 0}$ Wind Profilers}

In order to investigate the differences between the $w$ profiles and the $v_{D}$ profiles more thoroughly, we have simulated data not only for a single wind profiler but for an array of 400 wind profilers distributed evenly throughout the $9.4 \mathrm{~km}^{2}$ surface area of the LES model domain. The wind profilers are spaced by nine grid points $(144 \mathrm{~m})$ in the $x$ direction and by the same amount in the $y$ direction.

Figure 7 shows three vertical-velocity profiles. The solid line with no symbols is $\bar{w}(z)$, i.e., the vertical velocities from the LES, horizontally averaged over the whole model domain at selected heights. (Because of computing-time limitations, we did not compute the horizontal averages for every LES layer but 


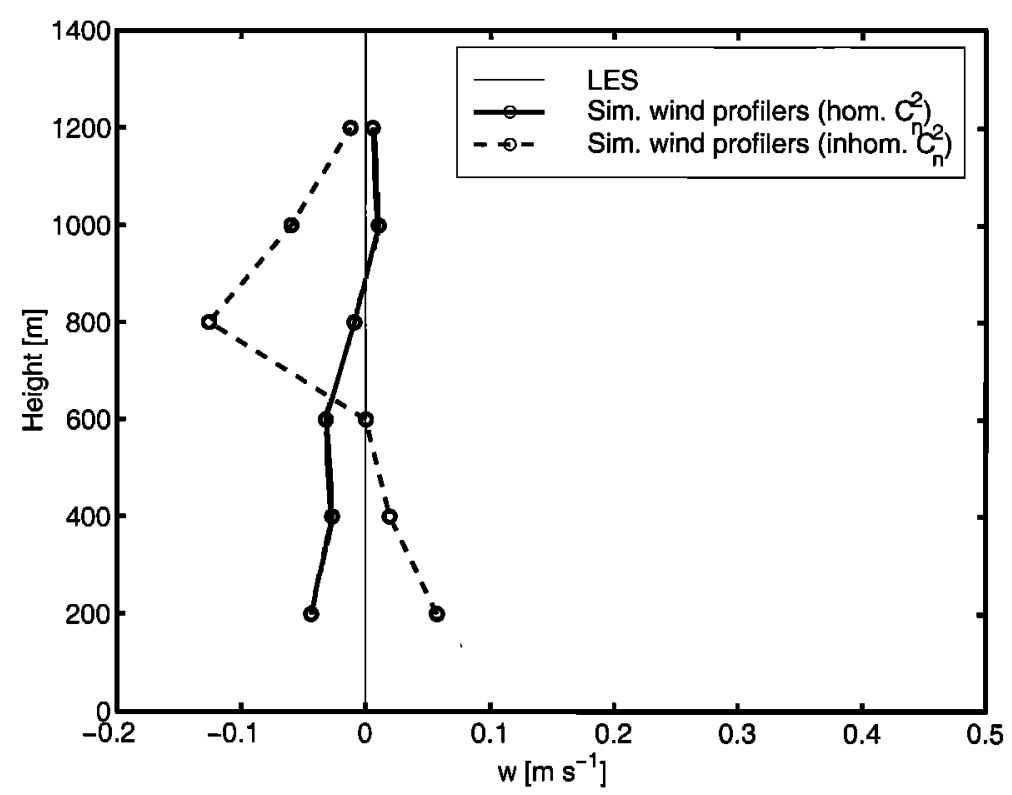

Figure 7. Vertical profiles of horizontally averaged simulated true and simulated observed vertical wind $(w)$ in a simulated, convective, atmospheric boundary layer, generated with a large-eddy simulation (LES) model. Plain solid line designates horizontal means of the LES-generated $w$ field (horizontally averaged, simulated true $w$ ); and solid and dotted lines with circles designate horizontal means of the simulated Doppler velocities (i.e., simulated observed mean $w$ ), obtained under the assumption of homogeneous and inhomogeneous $C_{n}^{2}$, respectively, within the radar resolution volumes.

only for every 25 th LES layer. This is the reason why the altitude spacing is $200 \mathrm{~m}$, in contrast to the vertical profiles for a single radar as shown in the previous section.) We have $\bar{w}(z)=0$ because the LES is set up with periodic boundary conditions in the horizontal directions; then continuity requires that the horizontally averaged, vertical wind be exactly zero. The two profiles with the circles are two vertical profiles of $\overline{v_{D}}$, i.e., the vertical profiles of the simulated Doppler velocities, averaged over the 400 radar sites. In other words, the $\overline{v_{D}}(z)$ profiles are horizontal averages of simulated wind-profiler observations of the vertical wind. The solid line with the circles is the $\overline{v_{D}}(z)$ profile obtained under the assumption of homogeneous $C_{n}^{2}$ within the radar resolution volumes, and the dotted line with the circles represents the $\overline{v_{D}}(z)$ profile obtained with the LES-generated, inhomogeneous $C_{n}^{2}$ distributions within the radar resolution volumes.

Figure 8 shows vertical profiles of the horizontalplane variances of the vertical wind. Again, the solid line with no symbols is the simulated truth as obtained directly from the LES; the two lines with the circles are the profiles of the variances of the Doppler velocities as obtained from the 400 simulated radars. As in Figure 7, the solid and dotted lines with circles represent the results obtained from the Dopplervelocity simulations made under the assumptions of homogeneous and inhomogeneous $C_{n}^{2}$, respectively. A fourth line (thin, with squares) represents the variances of the $W^{2}(\mathbf{r})$-weighted, LES-generated vertical velocities. As expected, this profile is almost identical to the profile of the $v_{D}$ variances obtained under the assumption of homogeneous $C_{n}^{2}$ (solid line with circles). Figure 8 shows that the wind-profiler measurements underestimate the vertical-wind variances. This bias is caused by the spatial filtering of the wind field over the radar's resolution volume. Typically, a significant portion of the wind variance is associated with length scales smaller than the size of the resolution volume. Therefore the bias is typically not negligible. It can be accounted for, however, on the basis of the spectral width, which is simultaneously measured and from which the nonresolved portion of the vertical-wind variance can be retrieved.

\section{Discussion}

As we have pointed out in section 1 , there are several mechanisms that can give rise to biases of wind-profiler velocity measurements. Part of the mo- 


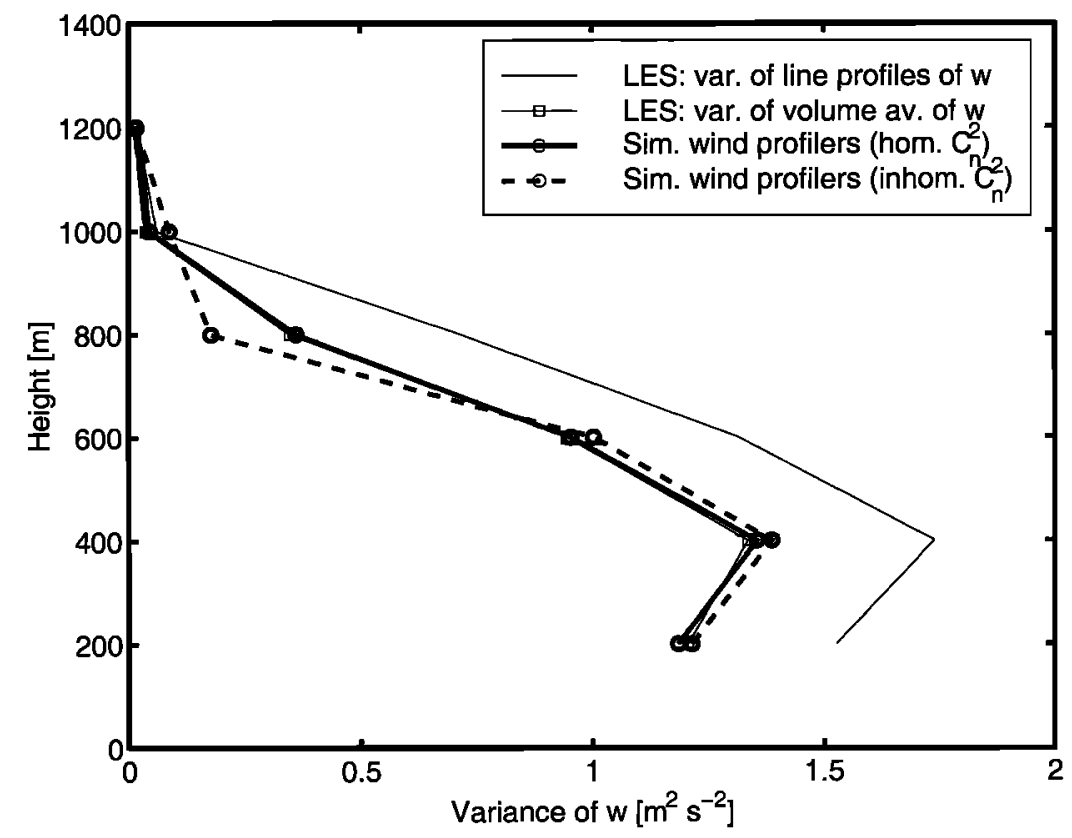

Figure 8. Vertical profiles of the variances of simulated true and simulated observed vertical wind within horizontal planes in a simulated convective atmospheric boundary layer, generated with a large-eddy simulation (LES) model. Plain solid line designates variances of the LES-generated $w$ field (simulated true $w$ variances); solid line with squares designates variances of the $W^{2}(\mathbf{r})$-weighted, LES-generated $w$ field; and solid and dotted lines with circles designate variance of the simulated Doppler velocities (i.e., simulated observed $w$ variances), obtained under the assumption of homogeneous and inhomogeneous $C_{n}^{2}$, respectively, within the radar resolution volumes.

tivation to develop the LES-based, radar-wind-profiler simulation technique presented in this paper was to extend the available methodology to identify, categorize, and quantify these biases.

In the previous section (see Figure 7) we have shown that the horizontal average of the vertical velocity measured by a simulated array of 400 wind profilers was not exactly zero (which was the simulated in situ truth as provided directly by the LES). There were deviations up to $5 \mathrm{~cm} \mathrm{~s}^{-1}$ in the simulation where we assumed uniform $C_{n}^{2}$ and deviations up to $13 \mathrm{~cm} \mathrm{~s}^{-1}$ in the simulation that was based on the $C_{n}^{2}$ field diagnostically obtained from the LES data set. Those deviations are of the order of the sampling error: We know that the $w$ variance $w^{2}$ is about $1 \mathrm{~m}^{2}$ $\mathrm{s}^{-2}$ in the CBL, and therefore the sampling error of the mean vertical velocity, $\sigma_{\bar{w}}$, is given by

$$
\sigma_{\bar{w}}=\sqrt{\frac{\overline{w^{2}}}{N}},
$$

where $N$ is the number of independent samples. That is, even if the 400 wind profilers would sample independently (i.e., even if there were no horizontal correlation between the vertical velocities observed at two points comparable to and larger than the windprofiler spacing of $144 \mathrm{~m}$ ), the sampling error would be as large as $\sigma_{\bar{w}}=5 \mathrm{~cm} \mathrm{~s}^{-1}$. The data point with a deviation of $13 \mathrm{~cm} \mathrm{~s}^{-1}$ from zero was obtained close to the inversion where the $C_{n}^{2}$ and its variance have a pronounced maximum, so that one can expect that a few very large $C_{n}^{2}$ values dominate the statistical ensemble.

Obviously, $\bar{w}$ as shown in Figure 7 varies smoothly with altitude. This is because the vertical-velocity fluctuations in a CBL are highly correlated in the vertical direction. Therefore it is to be expected that also the sampling errors in the vertical velocity are correlated in the vertical direction. Hence $\bar{w}$ varies smoothly with altitude.

We conclude that our simulations do not provide any support for the existence of a downward bias of up to $30 \mathrm{~cm} \mathrm{~s}^{-1}$ as reported by Angevine [1997]. Of course, this does not imply that such a bias does not exist. If it exists, however, it is not currently incorpo- 
rated in our LES code. In particular, we exclude a nonzero correlation between $C_{n}^{2}$ and $w$ as a candidate for causing this bias. Such a correlation would cause a $w$ bias of

$$
\Delta w(z)=\frac{\iint C_{n}^{2}(x, y, z) w(x, y, z) d x d y}{\iint C_{n}^{2}(x, y, z) d x d y},
$$

and from our LES data, we have found that the maximum of the magnitude $\Delta w(z)$ is located a few tens of meters above the ground and amounts to only a few millimeters per second. Apparently, the correlation between vertical velocity and radar reflectivity is much less important in the CBL than it is in the stably stratified atmosphere, as has been suggested by Nastrom and VanZandt [1994]. The bias, however, could still be large enough to be comparable to synoptic-scale vertical velocities.

\section{Summary and Conclusions}

In this paper we have presented a new technique to simulate clear-air, wind-profiler signals resulting from the backscattering of electromagnetic waves from clear-air refractive-index irregularities. This simulation technique is a combination of large-eddy simulation (LES) and the theory of radio wave propagation in turbulently mixed, clear air.

We analyzed wind-profiler observations simulated with that technique, and we did not find a significant vertical-velocity bias. This result is consistent with that of Angevine [1997], who presumes that the downward bias of up to $30 \mathrm{~cm} \mathrm{~s}^{-1}$ that he observed was caused by aerobiota (birds, insects). In general, our simulations support May et al. [1989], who have found that in the case of a good signal-to-noise ratio, it is mostly meteorological noise which determines the sampling error in standard wind-profiler observations, rather than any noise that stems from the measurement process itself.

In the future, the LES/radar-simulation technique could be applied to a huge variety of problems, from determining the effects of a finite signal-to-noise ratio, radio interference, ground clutter, and other contaminations on the retrieved meteorological quantities to problems concerning how to optimize the design of wind profilers and how to deploy and operate wind profilers in the field such that they provide maximum meteorological information.

\section{Appendix: Analysis of the Random Phase Model}

Here we derive in closed form the ensembleaveraged power spectrum of time series $I(t)$ obtained on the basis of (25) in the limit of an infinite number of realizations of the field of phases. The finiteness of the dwell time is taken into account.

The signal (25) can be written as

$$
I(t)=\sum_{p=1}^{N} a_{p} \exp \left(-j \omega_{p} t\right) \exp \left(-j \phi_{p}\right) \quad 0 \leq t \leq T_{d}
$$

where

$$
\begin{gathered}
a_{p}=A^{\prime} \sqrt{C_{n}^{2}\left(\mathbf{r}_{p}\right)}, \\
\phi_{p}=\varphi_{0}^{(p)}, \\
\omega_{p}=\mathbf{k}_{B}^{(p)} \cdot \mathbf{v}_{p} .
\end{gathered}
$$

Spectral representation of $I(t)$ should be a Fourier series because $I(t)$ is defined on a finite interval. The Fourier series representation of $\exp \left(-j \omega_{p} t\right)$ on the interval $0 \leq t \leq T_{d}$ is

$$
\exp \left(-j \omega_{p} t\right)=\sum_{n=-\infty}^{n=\infty} Z_{n p} \exp \left(-2 \pi j n t / T_{d}\right) .
$$

The frequencies of the Fourier series are $\omega_{n}=$ $2 \pi n / T_{d}$ for $n=\cdots,-2,-1,0,1,2, \cdots$, and the Fourier amplitudes are

$$
Z_{n p}=\frac{1-\exp \left[-j\left(\omega_{p}-\omega_{n}\right) T_{d}\right]}{j\left(\omega_{p}-\omega_{n}\right) T_{d}} .
$$

If for some integer $m$ we have $\omega_{p}=\omega_{m}$, then (A6) becomes $Z_{m p}=1$ and $Z_{n p}=0$ for $n \neq m$.

The spectrum of $I(t)$ for a collection of LES grid volumes at a single realization is

$$
\widetilde{S}\left(\omega_{n}\right)=\sum_{p^{\prime}=1}^{N} a_{p^{\prime}} Z_{n p^{\prime}}^{*} \exp \left(j \phi_{p^{\prime}}\right) \sum_{p=1}^{N} a_{p} Z_{n p} \exp \left(-j \phi_{p}\right) .
$$

The ensemble average of an infinite number of realizations $\widetilde{S}\left(\omega_{n}\right)$ is

$$
\begin{aligned}
S\left(\omega_{n}\right) & =\left\langle\tilde{S}\left(\omega_{n}\right)\right\rangle \\
& =\left\langle\sum_{p^{\prime}=1}^{N} a_{p^{\prime}} Z_{n p^{\prime}}^{*} \exp \left(j \phi_{p^{\prime}}\right) \sum_{p=1}^{N} a_{p} Z_{n p} \exp \left(-j \phi_{p}\right)\right\rangle,
\end{aligned}
$$


where the angle brackets denote the average over an ensemble of phases $\phi_{p}$ and $\phi_{p^{\prime}}$. Note that the $\omega_{n}$ are determined by the LES-generated $\mathbf{v}_{p}$ realization. Our random phase model is that the phases are assumed to be statistically independent unless, of course, $p^{\prime}=p$ :

$$
\begin{aligned}
& \left\langle\exp \left(j \phi_{p^{\prime}}-j \phi_{p}\right)\right\rangle \\
& \quad=\left\{\begin{array}{cc}
\left\langle\exp \left(j \phi_{p^{\prime}}\right)\right\rangle\left\langle\exp \left(-j \phi_{p}\right)\right\rangle=0 & p^{\prime} \neq p \\
\langle\exp (0)\rangle=1 & p^{\prime}=p
\end{array}\right\}=\delta_{p p^{\prime}} .
\end{aligned}
$$

Substitution into (A8) gives

$$
S\left(\omega_{n}\right)=\sum_{p=1}^{N} a_{p}^{2} Z_{n p} Z_{n p}^{*} .
$$

Now

$$
\begin{aligned}
& Z_{n p} Z_{n p}^{*} \\
& \quad=\left\{\begin{array}{cc}
\frac{2}{\left(\left(\omega_{p}-\omega_{n}\right) T_{d}\right)^{2}}\left[1-\cos \left(\left(\omega_{p}-\omega_{n}\right) T_{d}\right)\right] & \omega_{p} \neq \omega_{n} . \\
1 & \omega_{p}=\omega_{n}
\end{array}\right.
\end{aligned}
$$

A graph of (A10) versus $\omega_{n}$ is the Doppler spectral presentation. If $T_{d}$ is very large, then the $\omega_{n} \equiv$ $2 \pi n / T_{d}$ are very closely spaced such that the condition $\omega_{p}=\omega_{n}$ will be closely approximated for some $n$. In this case, $Z_{n p} Z_{n p}^{*}$ is approximately the Kronecker delta function, namely, $Z_{n p} Z_{n p}^{*} \simeq 1$ for $\omega_{p} \simeq$ $\omega_{n}$ and $Z_{n p} Z_{n p}^{*} \simeq 0$ otherwise. For this case or large $T_{d}$ the spectrum in (A11) is a weighted histogram of velocities with the weight $a_{p}^{2}$ assigned to each velocity component $\hat{\mathbf{u}} \cdot \mathbf{v}_{p}=\omega_{p} / k_{B}$, where $\hat{\mathbf{u}} \equiv \mathbf{k}_{B}^{(p)} / k_{B}$ is the unit vector in the direction of the Bragg wave number in the $p$ th subvolume. The graph of (A11) then takes on the appearance of a histogram if bins of frequency on the abscissa contain many values of $\omega_{n}$. It is thus clear that the Doppler spectrum is approximately a weighted histogram of radar-radial velocity components only if $T_{d}$ is very large.

One can calculate the standard deviation of the power spectrum for the random phase model. Using (A7) and (A8), we define the standard deviation of the power spectrum from individual realizations of the phases relative to the averaged power spectrum; namely, (A7) minus (A8) squared and averaged. Then take the square root, that is,

$$
\begin{aligned}
\sigma & \equiv\left\{\left\langle\left[\tilde{S}\left(\omega_{n}\right)-S\left(\omega_{n}\right)\right]^{2}\right\rangle\right\}^{1 / 2} \\
& =\left\{\left\langle\left[\left(\sum_{p^{\prime}=1}^{N} a_{p^{\prime}} Z_{n p^{\prime}}^{*} \exp \left(j \phi_{p^{\prime}}\right)\right.\right.\right.\right. \\
& \left.\left.\left.\left.\cdot \sum_{p=1}^{N} a_{p} Z_{n p} \exp \left(-j \phi_{p}\right)\right)-S\left(\omega_{n}\right)\right]^{2}\right\rangle\right\}^{1 / 2} \\
& =\left\{\left\langle\left[\sum_{p^{\prime}=1}^{N} a_{p^{\prime}} Z_{n p^{\prime}}^{*} \exp \left(j \phi_{p^{\prime}}\right)\right.\right.\right. \\
& \left.\left.\left.\cdot \sum_{p=1}^{N} a_{p} Z_{n p} \exp \left(-j \phi_{p}\right)\right]^{2}\right\rangle-\left[S\left(\omega_{n}\right)\right]^{2}\right\}^{1 / 2} \\
& =\sqrt{\left[\left(\sum_{p=1}^{N} \sum_{p^{\prime}=1}^{N} \sum_{p^{\prime \prime}=1}^{N} \sum_{p^{\prime \prime}=1}^{N} Q\left\langle s_{p}\right\rangle\right)-\left[S\left(\omega_{n}\right)\right]^{2}\right\}^{1 / 2}},
\end{aligned}
$$

where

$$
\begin{gathered}
s_{p}=\exp \left[j\left(-\phi_{p}+\phi_{p^{\prime}}-\phi_{p^{\prime \prime}}+\phi_{p^{\prime \prime}}\right)\right] \\
Q=a_{p} Z_{n p} a_{p^{\prime}} Z_{n p^{\prime}}^{*} a_{p^{\prime \prime}} Z_{n p^{\prime \prime}} a_{p^{m}} Z_{n p^{\prime \prime \prime}}^{*}
\end{gathered}
$$

The average of $s_{p}$ over uncorrelated phases gives $\delta_{p^{\prime} p} \delta_{p^{\prime \prime} p^{\prime \prime}}+\delta_{p^{\prime} p^{\prime \prime}} \delta_{p^{\prime \prime} p}$, substitution of which gives

$\sigma=\sqrt{2\left[S\left(\omega_{n}\right)\right]^{2}-\left[S\left(\omega_{n}\right)\right]^{2}}=\left|S\left(\omega_{n}\right)\right|=S\left(\omega_{n}\right)$.

That is, the random phase model has a standard deviation equal to the average power spectral level at each frequency $\omega_{n}$, independent of how long the dwell time is chosen. This is qualitatively confirmed by Figure 4 .

Acknowledgments. This study started during the time when the lead author (A.M.) worked as a visiting scientist at the Atmospheric Technology Division (ATD) of the National Center for Atmospheric Research (NCAR). A.M. appreciates the warm hospitality that he experienced during the 12 months at NCAR/ATD. His research stay was funded by the Deutsche Forschungsgemeinschaft (DFG). We thank David Carlson for approving a request for the computing resources needed to set up the LES for this study. A.M. appreciates interesting discussions with Rod Frehlich, Earl Gossard, Richard Lataitis, Valerian Tatarskii, and Dusan Zrnić. R.J.H.'s work was supported by National Science Foundation grant ATM-9618004. We are 
grateful to the three reviewers (K. S. Gage, P. T. May, and anonymous) who helped improve the paper.

\section{References}

Angevine, W. M., Errors in mean vertical velocities measured by boundary layer wind profilers, J. Atmos. Oceanic Technol., 14, 565-569, 1997.

Angevine, W. M., R. J. Doviak, and Z. Sorbjan, Remote sensing of vertical velocity variance and surface heat flux in a convective boundary layer, J. Appl. Meteorol., 33, 977-983, 1993.

Blackman, R. B., and J. W. Tukey, The Measurement of Power Spectra, Dover, Mineola, N. Y., 1958.

Chang, J. L., S. K. Avery, A. C. Riddle, S. E. Palo, and K. S. Gage, First results of tropospheric gravity wave momentum flux measurements over Christmas Island, Radio Sci., 32, 727-748, 1997.

Clifford, S. F., The classical theory of wave propagation in a turbulent medium, in Laser Beam Propagation in the Atmosphere, edited by W. Strohbehn, pp. 9-43, SpringerVerlag, New York, 1978.

Cohn, S. A., Radar measurements of turbulent eddy dissipation rate in the troposphere, J. Atmos. Oceanic Technol., 12, 85-95, 1995.

Deardorff, J. W., A numerical study of three-dimensional turbulent channel flow at large Reynolds numbers, $J$. Fluid Mech., 41, 453-480, 1970.

Deardorff, J. W., Stratocumulus-capped mixed layers derived from a three-dimensional model, Boundary Layer Meteorol., 18, 495-527, 1980.

Delage, D., R. Roca, F. Bertin, J. Delcourt, A. Crémieu, M. Massebeuf, and R. Ney, A consistency check of three radar methods for monitoring eddy diffusion and energy dissipation rates through the tropopause, Radio Sci., 32, 757-767, 1997.

Dörnbrack, A., Broadening of convective cells, Q. J. $R$. Meteorol. Soc., 123, 829-847, 1997.

Doviak, R. J., and D. S. Zrnić, Reflection and scatter formula for anisotropically turbulent air, Radio Sci., 19, 325-336, 1984.

Doviak, R. J., and D. S. Zrnić, Doppler Radar and Weather Observations, 2nd ed., Academic, San Diego, Calif., 1993.

Eaton, F. D., and G. D. Nastrom, Preliminary estimates of the vertical profiles of inner and outer scales from White Sands Missile Range, New Mexico, VHF radar observations, Radio Sci., 33, 895-903, 1998.

Frisch, A. S., and S. F. Clifford, A study of convection capped by a stable layer using Doppler radar and acoustic echo sounders, J. Atmos. Sci., 31, 1622-1628, 1974.

Gage, K. S., and J. L. Green, Evidence for specular reflection from monostatic VHF radar observations of the stratosphere, Radio Sci., 13, 991-1001, 1978.

Gage, K. S., and J. L. Green, A technique for determining the temperature profile from VHF radar observations, J. Appl. Meteorol., 21, 1146-1149, 1982.
Gage, K. S., J. L. Green, and T. E. VanZandt, Use of Doppler radar for the measurement of atmospheric turbulence parameters from the intensity of clear-air echoes, Radio Sci., 15, 407-416, 1980.

Galperin, B., and S. A. Orszag, Large Eddy Simulation of Complex Engineering and Geophysical Flows, Cambridge Univ. Press, New York, 1993.

Gossard, E. E., Radar research on the atmospheric boundary layer, in Radar in Meteorology, edited by D. Atlas, pp. 477-527, Am. Meteorol. Soc., Boston, Mass., 1990.

Gossard, E. E., and R. G. Strauch, Radar Observations of Clear Air and Clouds, Elsevier, New York, 1983.

Gossard, E. E., D. E. Wolfe, K. P. Moran, R. A. Paulus, K. D. Anderson, and L. T. Rogers, Measurements of clear-air gradients and turbulence properties with radar wind profilers, J. Atmos. Oceanic Technol., 15, 321-342, 1998.

Gossard, E. E., D. E. Wolfe, and B. B. Stankov, Measurements of humidity profiles in the atmosphere by the Global Positioning System and radar wind profilers, $J$. Atmos. Oceanic Technol., 16, 156-164, 1999.

Hocking, W. K., On the extraction of atmospheric turbulence parameters from radar backscatter Doppler spectra, 1, Theory, J. Atmos. Terr. Phys., 45, 89-102, 1983.

Hocking, W. K., R. Rüster, and P. Czechowsky, Absolute reflectivities and aspect sensitivities of VHF radio scatterers measured with the SOUSY radar, J. Atmos. Terr. Phys., 48, 131-144, 1986.

Holdsworth, D. A., and I. M. Reid, A simple model of atmospheric radar backscatter: Description and application to the full correlation analysis of spaced antenna data, Radio Sci., 30, 1263-1280, 1995.

Hoppe, U.-P., and D. C. Fritts, High-resolution measurements of vertical velocity with the European incoherent scatter VHF radar, 1, Motion field characteristics and measurement biases, J. Geophys. Res., 100(D8), 16,81316,825, 1995.

Jenkins, G. M., and D. G. Watts, Spectral Analysis and Its Applications, Holden-Day, Merrifield, Va., 1968.

Kaltenbach, H.-J., T. Gerz, and U. Schumann, Large-eddy simulation of homogeneous turbulence and diffusion in stably stratified shear flow, J. Fluid Mech., 280, 1-40, 1994.

Leonard, A., Energy cascade in large eddy simulations of turbulent fluid flows, $A d v$. Geophys., 18A, 237-248, 1974.

Lilly, D. K., The representation of small-scale turbulence in numerical simulation experiments, in Proceedings of the IBM Scientific Computing Symposium on Environmental Science, pp. 195-210, Thomas J. Watson Res. Cent., Yorktown Heights, N. Y., 1967.

Lippmann, J., M. Bauer, and G. Peters, Methods of virtual heat flux determination from boundary layer wind profiler/RASS measurements, Contrib. Atmos. Phys., 69, 119-128, 1997. 
Mason, P. J., Large-eddy simulation: A critical review of the technique, Q. J. R. Meteorol. Soc., 120, 1-26, 1994.

Mason, P. J., and A. R. Brown, On subgrid models and filter operations in large eddy simulations, J. Atmos. Sci., 56, 2101-2114, 1999.

May, P. T., T. Sato, M. Yamamoto, S. Kato, T. Tsuda, and S. Fukao, Errors in the determination of wind speed by Doppler radar, J. Atmos. Oceanic Technol., 6, 235-242, 1989.

Muschinski, A., A similarity theory of locally homogeneous and isotropic turbulence generated by a Smagorinskytype LES, J. Fluid Mech., 325, 239-260, 1996a.

Muschinski, A., Possible effect of Kelvin-Helmholtz instability on VHF radar observations of the mean vertical wind, J. Appl. Meteorol., 35, 2210-2217, 1996b.

Muschinski, A., Turbulence and gravity waves in the vicinity of a midtropospheric warm front: A case study using VHF echo-intensity measurements and radiosonde data, Radio Sci., 32, 1161-1178, 1997.

Muschinski, A., The first moments of the variance- and cross-spectra of standard and interferometric clear-air Doppler-radar signals, NCAR Tech. Note $441+S T R$, Natl. Cent. for Atmos. Res., Boulder, Colo., 1998.

Nastrom, G. D., and T. E. VanZandt, Mean vertical motion seen by radar wind profilers, J. Appl. Meteorol., 33, 984-995, 1994.

Peltier, L. J., and J. C. Wyngaard, Structure-function parameters in the convective boundary layer from large-eddy simulation, J. Atmos. Sci., 52, 3641-3660, 1995.

Rao, D. N., P. Kishore, T. N. Rao, V. B. Rao, K. K. Reddy, M. Yarraiah, and M. Hareesh, Studies on refractivity structure constant, eddy dissipation rate, and momentum flux at a tropical latitude, Radio Sci., 32, 1375-1389, 1997.

Röttger, J., and C. H. Liu, Partial reflection and scattering of VHF radar signals from the clear atmosphere, Geophys. Res. Lett., 5, 357-360, 1978.

Schmidt, H., and U. Schumann, Coherent structure of the convective boundary layer derived from large-eddy simulations, J. Fluid Mech., 200, 511-562, 1989.

Sheppard, E. L., and M. F. Larsen, Analysis of model simulations of spaced antenna/radar interferometer measurements, Radio Sci., 27, 759-768, 1992.

Smith, T. L., and S. G. Benjamin, Impact of network wind profiler data on a 3-h data assimilation system, Bull. Am. Meteorol. Soc., 74, 801-807, 1993.

Sullivan, P. P., J. C. McWilliams, and C.-H. Moeng, A grid nesting method for large-eddy simulation of planetary boundary-layer flows, Boundary Layer Meteorol., 80, 167202, 1996.

Sullivan, P. P., C.-H. Moeng, B. Stevens, D. H. Lenschow, and S. D. Mayor, Structure of entrainment zone capping the convective atmospheric boundary layer, J. Atmos. Sci., 55, 3042-3064, 1998.

Tatarskii, V. I., Wave Propagation in a Turbulent Medium, McGraw-Hill, New York, 1961.

Taylor, G. I., The spectrum of turbulence, Proc. $R$. Soc. London, Ser. A, 164, 476-490, 1938.

VanZandt, T. E., J. L. Green, K. S. Gage, and W. L. Clark, Vertical profiles of refractivity turbulence structure constant: Comparison of observations by the Sunset Radar with a new theoretical model, Radio Sci., 13, 819-829, 1978.

Vincent, R. A., and I. M. Reid, HF doppler measurements of mesospheric gravity wave momentum fluxes, J. Atmos. Sci., 40, 1321-1333, 1983.

Weinstock, J., Using radar to estimate dissipation rates in thin layers of turbulence, Radio Sci., 16, 1401-1406, 1981.

Wyngaard, J. C., L. J. Peltier, and S. Khanna, LES in the surface layer: Surface fluxes, scaling, and SGS modeling, J. Atmos. Sci., 55, 1733-1754, 1998.

Yaglom, A. M., Laws of small-scale turbulence in atmosphere and ocean (in commemoration of the 40th anniversary of the theory of locally isotropic turbulence), Izv. Russ. Acad. Sci. Atmos. Oceanic Phys., Engl. Transl., 17, 919-935, 1981.

Zrnić, D. S., Simulation of weatherlike Doppler spectra and signals, J. Appl. Meteorol., 14, 619-620, 1975.

S. A. Cohn, NCAR Atmospheric Technology Division, 3450 Mitchell Lane, Boulder, CO 80307.

R. J. Doviak, NOAA National Severe Storms Laboratory, 1313 Halley Circle, Norman, OK 73069.

R. J. Hill and D. B. Wuertz, NOAA Environmental Technology Laboratory, 325 Broadway, R/E/ET4, Boulder, CO 80303-3328.

D. H. Lenschow and P. P. Sullivan, NCAR Mesoscale and Microscale Meteorology Division, 3450 Mitchell Lane, Boulder, CO 80307.

A. Muschinski, CIRES, University of Colorado/NOAA, and NOAA Environmental Technology Laboratory, 325 Broadway, R/E/ET4, Boulder, CO 80303-3328. (amuschinski@etl. noaa.gov)

(Received March 11, 1999; revised August 9, 1999; accepted August 10, 1999.) 